Check for updates
OPEN ACCESS

\section{Dietary alteration of n-3 and n- 6 fatty acids for headache reduction in adults with migraine: randomized controlled trial}

\author{
Christopher E Ramsden, ${ }^{1,2,3}$ Daisy Zamora, ${ }^{1,4}$ Keturah R Faurot, ${ }^{3}$ Beth MacIntosh, ${ }^{3,5}$ \\ Mark Horowitz, ${ }^{1}$ Gregory S Keyes, ${ }^{1}$ Zhi-Xin Yuan, ${ }^{1}$ Vanessa Miller, ${ }^{3}$ Chanee Lynch, ${ }^{3}$ \\ Gilson Honvoh, ${ }^{3,6}$ Jinyoung Park, ${ }^{3}$ Russell Levy, ${ }^{7}$ Anthony F Domenichiello, ${ }^{1}$ Angela Johnston, ${ }^{3}$ \\ Sharon Majchrzak-Hong, ${ }^{2}$ Joseph R Hibbeln, ${ }^{2}$ David A Barrow, ${ }^{7}$ James Loewke, ${ }^{2}$ John M Davis, ${ }^{4}$ \\ Andrew Mannes, ${ }^{8}$ Olafur S Palsson, ${ }^{6}$ Chirayath M Suchindran, ${ }^{9}$ Susan A Gaylord, ${ }^{3}$ \\ J Douglas Mann ${ }^{10}$
}

For numbered affiliations see end of the article

Correspondence to: C Ramsden Lipid Peroxidation Unit, Laboratory of Clinical Investigation, National Institute on Aging, NIH 251 Bayview Blvd, Baltimore, MD, USA chris.ramsden@nih.gov (ORCID 0000-0002-4058-7630) Additional material is published online only. To view please visit the journal online.

Cite this as: $B M J$ 2021;374:n1448 http://dx.doi.org/10.1136/bmj.n1448 Accepted: 27 May 2021

\section{ABSTRACT}

\section{OBJECTIVE}

To determine whether dietary interventions that increase $n-3$ fatty acids with and without reduction in n-6 linoleic acid can alter circulating lipid mediators implicated in headache pathogenesis, and decrease headache in adults with migraine.

\section{DESIGN}

Three arm, parallel group, randomized, modified double blind, controlled trial.

\section{SETTING}

Ambulatory, academic medical center in the United States over 16 weeks.

\section{PARTICIPANTS}

182 participants ( $88 \%$ women, mean age 38 years) with migraines on $5-20$ days per month (67\% met criteria for chronic migraine).

\section{INTERVENTIONS}

Three diets designed with eicosapentaenoic acid (EPA), docosahexaenoic acid (DHA), and linoleic acid altered as controlled variables: $\mathrm{H} 3$ diet $(n=61)$ increase EPA+DHA to $1.5 \mathrm{~g} /$ day and maintain linoleic acid at around $7 \%$ of energy; H3-L6 diet $(n=61)-$ increase $\mathrm{n}-3 \mathrm{EPA}+\mathrm{DHA}$ to $1.5 \mathrm{~g} /$ day and decrease linoleic acid to $\leq 1.8 \%$ of energy; control diet $(n=60)-$ maintain EPA+DHA at $<150 \mathrm{mg} /$ day and linoleic acid at around $7 \%$ of energy. All participants received foods accounting for two thirds of daily food energy and continued usual care.

\section{WHAT IS ALREADY KNOWN ON THIS TOPIC}

Modern industrialized diets tend to be low in $n-3$ eicosapentaenoic acid (EPA) and docosahexaenoic acid (DHA), and high in n-6 linoleic acid

These fatty acids serve as precursors to endogenous signaling molecules (oxylipins) shown to increase (linoleic acid) or decrease (EPA+DHA) pain in preclinical models

\section{WHAT THIS STUDY ADDS}

Adults with chronic and episodic migraine in the United States were randomized to the H3 diet (increased EPA+DHA), the H3-L6 diet (increased EPA+DHA, reduced linoleic acid), or a control diet (average US intake of n-3 and n- 6 fatty acids) While the benefit of the $\mathrm{H} 3$ and H3-L6 diets on quality of life was inconclusive, the reduction in headaches was robust, particularly for the H3-L6 diet

Both active diets altered blood levels of bioactive oxylipins implicated in headache pathogenesis in a manner consistent with a lowered nociceptive state, and did not alter classic mediators of headache pain (prostaglandins, calcitonin gene related peptide)

\section{MAIN OUTCOME MEASURES}

The primary endpoints (week 16) were the antinociceptive mediator 17-hydroxydocosahexaenoic acid (17-HDHA) in blood and the headache impact test (HIT-6), a six item questionnaire assessing headache impact on quality of life. Headache frequency was assessed daily with an electronic diary.

\section{RESULTS}

In intention-to-treat analyses ( $n=182)$, the H3-L6 and $\mathrm{H} 3$ diets increased circulating 17-HDHA (log $\mathrm{ng} / \mathrm{mL}$ ) compared with the control diet (baselineadjusted mean difference $0.6,95 \%$ confidence interval 0.2 to $0.9 ; 0.7,0.4$ to 1.1 , respectively). The observed improvement in HIT-6 scores in the H3-L6 and $\mathrm{H} 3$ groups was not statistically significant $(-1.6$, -4.2 to 1.0 , and $-1.5,-4.2$ to 1.2 , respectively). Compared with the control diet, the $\mathrm{H} 3-\mathrm{L} 6$ and $\mathrm{H} 3$ diets decreased total headache hours per day $(-1.7$, -2.5 to -0.9 , and $-1.3,-2.1$ to -0.5 , respectively), moderate to severe headache hours per day $(-0.8$, -1.2 to -0.4 , and $-0.7,-1.1$ to -0.3 , respectively), and headache days per month $(-4.0,-5.2$ to -2.7 , and $-2.0,-3.3$ to -0.7 , respectively). The H3-L6 diet decreased headache days per month more than the $\mathrm{H} 3$ diet $(-2.0,-3.2$ to -0.8$)$, suggesting additional benefit from lowering dietary linoleic acid. The H3$\mathrm{L} 6$ and $\mathrm{H} 3$ diets altered $\mathrm{n}-3$ and $\mathrm{n}-6$ fatty acids and several of their nociceptive oxylipin derivatives in plasma, serum, erythrocytes or immune cells, but did not alter classic headache mediators calcitonin gene related peptide and prostaglandin E2.

\section{CONCLUSIONS}

The $\mathrm{H} 3-\mathrm{L} 6$ and $\mathrm{H} 3$ interventions altered bioactive mediators implicated in headache pathogenesis and decreased frequency and severity of headaches, but did not significantly improve quality of life.

\section{TRIAL REGISTRATION}

ClinicalTrials.gov NCT02012790

\section{Introduction}

Migraine is among the largest causes of disability worldwide. $^{1-3}$ Although treatment options for migraine have improved in the last decade, many patients continue to experience substantial pain and disability despite taking multiple drugs. As with many multifactorial chronic diseases, an incomplete understanding of the underlying biological mechanisms has limited development of effective approaches for prevention and treatment. 
A role for dietary $n-3$ and $n-6$ fatty acids in migraine pathogenesis

n-3 and n- 6 fatty acids are major components of tissues implicated in migraine pathogenesis ${ }^{4-6}$ where they serve as precursors for several families of bioactive lipid mediators that regulate pain (eg, prostaglandins, leukotrienes, resolvins, maresins). ${ }^{7}$ These lipid mediators are collectively known as oxylipins. Because humans cannot synthesize n- 3 and n- 6 fatty acids de novo, the levels of these fatty acids and their oxylipin derivatives can be altered by diet (fig 1)..$^{56-11}$ Several families of oxylipin receptors are enriched in trigeminal nerve endings ${ }^{12}$ and central pain processing pathways, ${ }^{13}$ where they regulate sensitization and the release of the headache related neuropeptide calcitonin gene related peptide ${ }^{12}$; this implies a direct link between n-3 and n- 6 fatty acids and headache pathogenesis. Several oxylipins derived from n- 6 fatty acids have pronociceptive (pain promoting) properties. ${ }^{14-20}$ For example, infusion of prostaglandins derived from $\mathrm{n}-6$ arachidonic acid evokes migraine like attacks in humans. ${ }^{21} 22$ Oxylipin derivatives of n-6 linoleic acid sensitize trigeminal nerve endings ${ }^{23}$ and evoke behavioral pain responses in preclinical models. ${ }^{16182425}$ By contrast, several oxylipins derived from n-3 eicosapentaenoic acid (EPA) and docosahexaenoic acid (DHA) have potent antinociceptive (pain reducing) properties. ${ }^{26-29}$ Most notably, 17-hydroxydocosahexaeonic acid (17HDHA) is the pathway precursor for several families of oxylipins reported to have potent antinociceptive effects in experimental models, ${ }^{29-38}$ and has been linked to lower pain scores in patients with arthritis. ${ }^{39}$

We previously showed that targeted dietary manipulation of precursor fatty acids can alter oxylipins in tissues associated with migraine headaches and other idiopathic pain syndromes in rodents. ${ }^{51040}$ Further, in a pilot trial of 67 adults with chronic daily headache, we showed that a 12 week, food based intervention that increased dietary n-3 EPA+DHA while decreasing n-6 linoleic acid reduced headache days per month compared with an intervention that decreased linoleic acid alone. ${ }^{6} 8114142$ This study provided proof of concept that food based increases in n-3 EPA+DHA with concurrent reduction in n- 6 linoleic acid can be effective for headache. However, in this pilot trial both arms lowered linoleic acid to about $2.5 \%$ of calories, which is less than half of the amount in most modern diets. ${ }^{43} 44$ Questions remained about whether dietary interventions targeting n-3 EPA+DHA and n-6 linoleic acid can be a useful adjunct pain treatment compared with diets that contain amounts of these fatty acids that are typical of modern industrialized diets; and whether the combination of increasing n-3 EPA+DHA and decreasing n- 6 linoleic acid produces maximal pain reduction or whether the same effect can be achieved with n-3 EPA+DHA alone.

We investigate the biochemical and clinical effects of two active diets (one increasing n-3 EPA+DHA alone (H3) and one with concurrent reduction in n-6 linoleic acid (H3-L6)) compared with a control diet containing average amounts (in the United States) of n-3 EPA+DHA and n- 6 linoleic acid in adults with chronic and episodic migraine. We hypothesized that both active diets would increase circulating 17-HDHA and alter other antinociceptive oxylipins in a manner that would decrease the frequency and severity of headache pain and therefore improve headache impact on quality of life. We further hypothesized that the H3-L6 diet would produce more pronounced biochemical alterations and pain reduction compared with the $\mathrm{H} 3$ diet.

\section{Materials and methods}

The trial protocol has been previously published. ${ }^{45}$ We recruited adults with migraine on 5-20 days per month and meeting the 2004 International Classification of Headache Disorders criteria for migraine, with or without aura. A board certified headache neurologist (JDM) certified that patients met the diagnosis of migraine with or without aura after a headache history and exam. Other inclusion criteria included having a headache history for at least two years, being under the care of a physician for headache management, having internet access, ability to communicate in English, and willingness to comply with study procedures, including maintaining an online daily headache diary. Exclusions included pregnancy or breastfeeding, recent changes in use of female hormones, major head trauma or surgery of the head or neck within three years of screening, and use of fish oil or omega 3 fatty acids as supplements. Table S1 lists complete inclusion and exclusion criteria.

The trial was conducted at the University of North Carolina at Chapel Hill from July 2014 to May 2018. Initially, the study was designed to recruit participants with episodic migraine (8-14 migraine days per month). After the study began and 32 participants had already been randomized (May 2015), we expanded this criterion to 5-20 migraine days per month. This change was made out of concern that enrollment targets might not be met with the more stringent criteria. All protocol changes were approved by the funding agency (National Center for Complementary and Integrative Health, National Institutes of Health).

After the nature of the trial was explained and written informed consent was obtained, participants were advised to continue seeing their regular headache physician for usual care throughout the trial. Participants recorded headaches and drug use in an online daily headache diary for at least four weeks (preintervention run-in phase). Participants who completed at least $80 \%$ of the diary entries during this time and met the migraine days per month criterion were randomly assigned to one of three dietary interventions to be maintained for 16 weeks. Laboratory analyses of precursor fatty acids, oxylipins, and neuropeptides were completed from January 2019 to February 2020. Trial procedures were approved by the University of North Carolina at Chapel Hill institutional review board (13-3284) and registered under ClinicalTrials.gov (NCT02012790). 


\section{Dietary n-3 and n-6 fatty acids are incorporated into blood and delivered to headache relevant tissues}
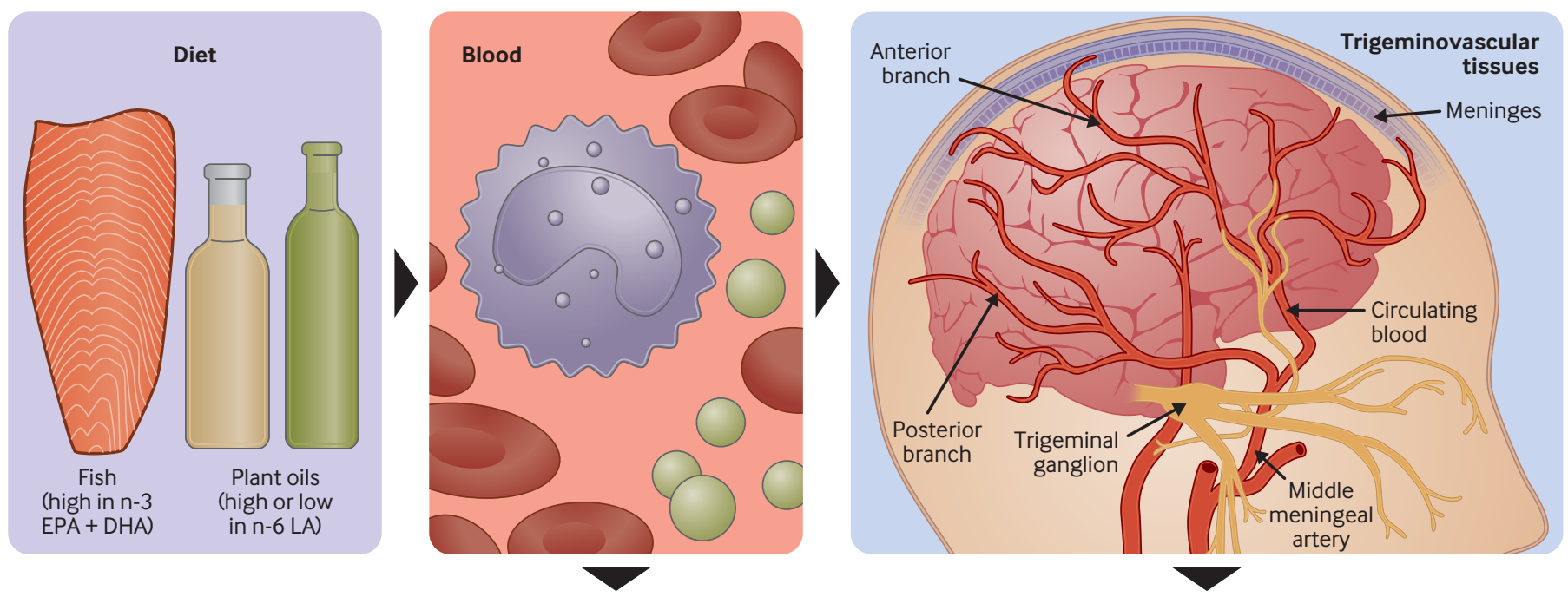

n-3 and n-6 fatty acids are enzymatically converted to signaling molecules (oxylipins) that regulate pain

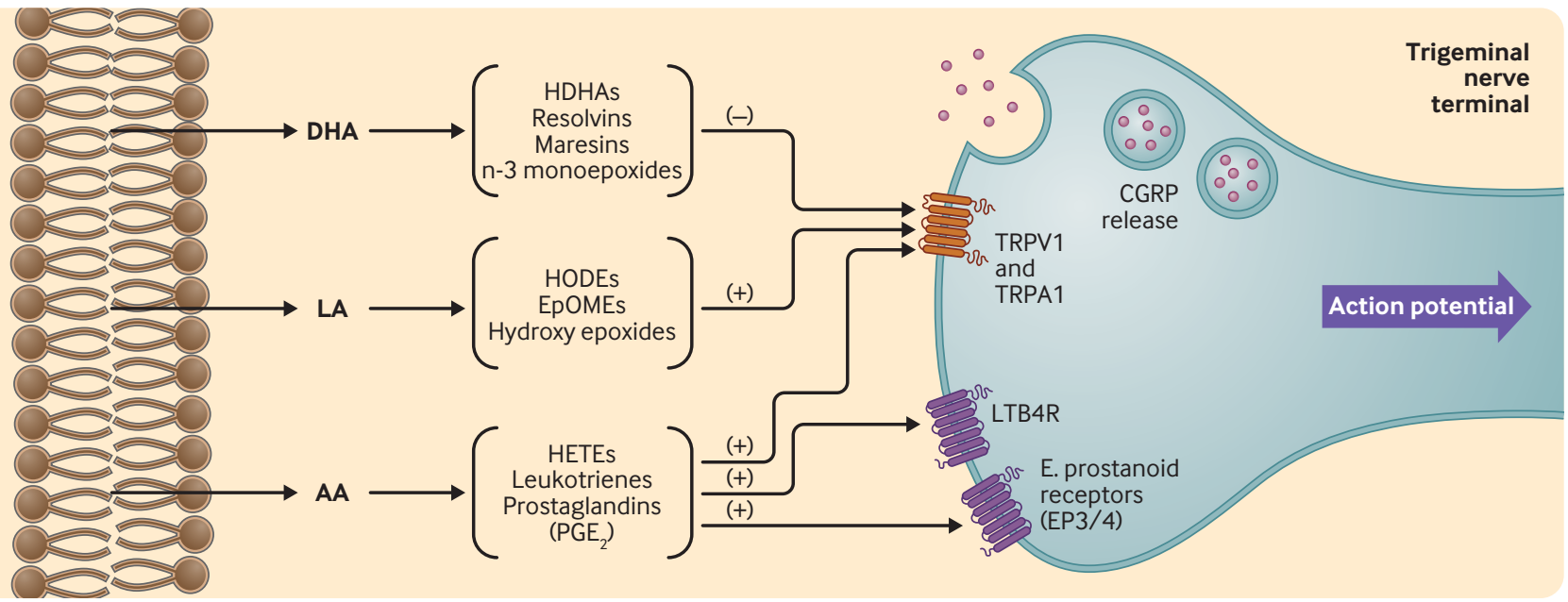

Fig 1 | Proposed mechanisms linking dietary n-6 and n-3 fatty acids and their oxylipin derivatives to headache pathogenesis. Upper left panel: modern industrialized diets tend to be low in food sources of n-3 EPA and DHA (eg, finfish and shellfish) and high in vegetable oils rich in n- 6 LA; humans cannot synthesize n-3 and n-6 fatty acids de novo, and so dietary intakes affect their abundance in blood and tissues; upper middle panel: circulating blood contains pools of n-3 and n-6 fatty acids, including red blood cells which serve as a proxy for tissue fatty acids, and immune cells which can infiltrate inflamed tissues and are direct precursors to nociceptive oxylipins that can act on nerve endings; upper right panel: $n-3$ and $n-6$ fatty acids are major components of lipid membranes of tissues innervated by the trigeminal nerve, including meninges, cranial vessels, mucosal membranes, the trigeminal nerve itself, and the lipid pools in pain processing pathways (myelin, astrocytes, and central nervous system neurons); lower panel: these lipid pools serve as precursors for synthesis of oxylipins with pronociceptive and antinociceptive properties, including prostaglandins, leukotrienes, octadecanoids, resolvins, and n-3 monoepoxides. Oxylipins activate GPCRs and TRP channel receptors to regulate trigeminal nerve activation and localized release of CGRP. Oxylipins also act directly on vascular tissues to evoke vasodilation and increase vascular permeability (not shown). Four oxylipin receptors that are enriched in human trigeminal nerve are shown in the lower panel. $A A=a r a c h i d o n i c ~ a c i d ;$

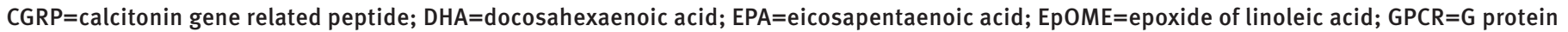
coupled receptor; HDHA=hydroxydocosahexaenoic acid; HETE=hydroxyeicosatetraenoic acid; HODE=hydroxyoctadecadienoic acid; LA=linoleic acid; LTB4R=leukotriene B4 receptor; TRP=transient receptor potential

\section{Randomization and masking}

Participants were randomized to one of the three diets during their first visit with the dietitian. At that time, the dietitian accessed an online interface that used an algorithm with permuted block design and random number sequence, ensuring concealed participant allocation. A modified double blind design was used with only the dietitian unmasked at randomization, which was needed to counsel participants in their assigned diets. All other investigators, study and laboratory staff, and each participant's personal physician were masked to group assignment for the duration of the trial. Participants received counseling, food provision, and website access in accordance with assigned interventions and were masked to the nature of the other interventions.

Diet interventions and nutrient intake assessment The three diets were high n-3 (H3 diet), high n-3+low n-6 (H3-L6 diet), and average US n-3 and n-6 intake 
(control diet). ${ }^{4546}$ The $\mathrm{H} 3$ diet was designed to increase $\mathrm{EPA}+\mathrm{DHA}$ to $1.5 \mathrm{~g} /$ day while maintaining average US intakes of linoleic acid ( $7.2 \%$ of energy). The H3-L6 diet was designed to increase n-3 EPA+DHA to $1.5 \mathrm{~g} /$ day and concurrently decrease n- 6 linoleic acid to $\leq 1.8 \%$ of energy. The control diet was designed to maintain average US intakes of EPA+DHA $(<150 \mathrm{mg} /$ day) and linoleic acid (7.2\% of energy). All three diets were designed to be as similar as possible, with the differences being the study provided oil and butter formulations, and the protein foods provided to achieve the fatty acid targets (eg, high fat fish for the H3 and H3-L6 groups $v$ low fat fish or poultry for the control group). The H3-L6 diet was designed to have linoleic acid modified as a controlled variable through isocaloric replacement of linoleic acid with fats that are not enzymatically converted to oxidized lipid mediators (mostly monounsaturated fats and some saturated fats). This modification was achieved by giving the $\mathrm{H} 3$ and control groups a blend of corn oil and extra virgin olive oil and a blend of butter and corn oil, whereas the H3-L6 group was given a blend of macadamia nut oil and extra virgin olive oil and regular butter. Participants in all groups received the same instruction about oils (to prepare foods at home as often as possible with only the study provided oils or butter, and when grocery shopping or dining out to avoid all other oils except olive oil, butter, palm oil, and coconut oil). All three diets were designed to be eucaloric (not promote weight loss). The supplement (pages 3-5) gives a description of the interventions, including foods provided and sample meal plan.

The diets were designed to be equally credible and acceptable with equivalent intensity and amounts of dietitian counseling, intervention materials, study foods and oils, and macronutrient and energy intake. Intensive dietitian counseling was provided at randomization and at 2-3 week intervals throughout the 16 week intervention phase. Foods that met nutrient targets, adequate for two meals and two snacks per day, were provided at each diet counseling session. To maximize credibility, all study oils contained $25 \%$ extra virgin olive oil, which provided an equivalent green hue; the control group received substantial amounts of fish and shellfish products that were low in EPA+DHA, including a low EPA+DHA tuna fish; all three diet groups received identical amounts of packaged foods (eg, frozen fruits and vegetables, whole wheat bread, low fat cheese) and frozen proteins (eg, fish and lean poultry); and all three groups had access to equally intensive, intervention specific web materials designed to complement food provision and diet advice. Nutrient intakes were assessed using two unannounced, telephone administered, 24 hour recalls (one weekday, one weekend day) during each phase (preintervention and intervention), as previously described. ${ }^{45} 46$

Expectation of Benefit/Credibility Questionnaire The Expectation of Benefit/Credibility Questionnaire is based on a validated scale developed for assessment of rationale credibility and treatment expectancy in clinical outcome studies. ${ }^{47-49}$ The questionnaire consists of five questions, each rated from 0 to 9 on a Likert scale, with higher scores indicating that participants find an intervention believable, logical, and possibly successful at reducing headache (supplement, page 6). The questionnaire was administered at the end of the randomization visit, after the first dietitian counselling session, and before controlled provision of study foods and oils.

\section{Headache related clinical endpoints}

The primary clinical endpoint was the six item headache impact test (HIT-6), ${ }^{50}$ a self-reported questionnaire measuring the "impact that headaches have on the ability to function on the job, at school, at home, and in social situations". ${ }^{51}$ A sample question in the HIT- 6 is, "In the past 4 weeks, how often have you felt fed up or irritated because of your headaches?" The scale ranges from 36 to 78 points. A higher score indicates a greater impact of headaches on quality of life. The between group minimally important difference in HIT-6 score has been estimated at 1.5 points in a primary care population of people with migraine. ${ }^{52}$ This corresponds to a "somewhat better" perceived clinical improvement after three months compared with "about the same". Participants completed the HIT- 6 before randomization and at the 16 week followup visit.

During the preintervention and intervention phases, participants kept a headache diary that was available on a secure website through a computer or smart phone interface. Participants were given a template on which to enter headache status for each hour of the day, with the options being mild, moderate, severe, or sleeping (sample in supplement, pages 7-9). ${ }^{64}$ Participants also recorded acute drugs used for headache and the number of doses per day. If a participant did not complete the diary for a given day, a text or email reminder was sent the following day. To limit recall bias, participants were only able to enter information for the current and previous calendar day. Variables derived from the headache diary include the number of total headache hours per day (any intensity) and the number of headache days per month (number of days in the last month in which at least an hour of headache of any intensity was experienced). The number of moderate to severe headache hours per day was defined as pain requiring drugs every 3-4 hours, ability to function less than $80 \%$, mild to severe nausea or sensitivity to light or sound, and pain rating of 4 or greater on a scale of $1-10(1=$ minimal and $10=$ worst pain ever). The number of instances per day of drug use for acute pain was calculated based on the number of doses participants reported consuming for a particular drug. Categories of acute pain drugs included nonsteroidal anti-inflammatory drugs (NSAIDs) or aspirin, triptans, and other (eg, acetaminophen, opioids). Ergotamines were not considered a separate category because only a single mention of use (one participant, one day) was made during the trial. 
Overuse of acute drugs was operationalized based on the International Classification of Headache Disorders (third edition) definitions for drug overuse headache, ${ }^{53}$ and calculated from the number of days participants reported drug use in the headache diary in the month before randomization, and in the last month of the intervention. For example, acute pain drug overuse was defined as consumption for $\geq 10$ days per month of triptans, opioids, combination analgesics, or multiple drug classes not individually overused, or consumption for $\geq 15$ days per month of acetaminophen, NSAIDs or aspirin, other non-opioid analgesics, or non-opioid analgesics with no single class overused.

\section{Preventive drugs}

The trial was envisioned as an adjunct to usual care for migraine, therefore people with migraine were not excluded based on their use of drugs for migraine treatment, including botulinum toxin. Moreover, changes in preventive drugs were allowed during the study. At baseline, participants provided a drug history that was reviewed with the study neurologist. For preventive drugs, any changes were recorded at visits for weeks 4, 10, and 16 (use of acute drugs was recorded in the electronic headache diary). Table S2 describes categories of preventive drugs commonly used in the treatment of migraine (anticonvulsants, $\beta$ blockers or verapamil, antidepressants, botulinum toxin).

\section{Adverse events}

Adverse events were assessed at each visit. Participants were asked specifically about rash, tissue swelling, shortness of breath, swollen tongue, fatigue, and weight change of more than $1.4 \mathrm{~kg}$ in two weeks, blood draw site swelling and pain, other new symptoms, or worsening of previous symptoms. Details of any event were requested and the participant was followed until the event resolved. All potential adverse events were reported to the study coordinator and reviewed with the principal investigator. In addition to assessing events during study visits, the electronic headache diary comments were monitored by the study team. The study coordinator or research assistant followed up on comments that could be interpreted as an adverse event, including headache symptoms unusual for the participant.

\section{Specimen collection, processing, and biochemical analyses}

Detailed methods used for these biochemical analyses have been previously published. ${ }^{6} 54$ Briefly, fasting blood was collected and immediately processed to plasma, serum, and erythrocytes at the conclusion of the run-in phase, and after 4, 10, and 16 weeks of diet exposure, as previously described. ${ }^{45}$ Fasting blood was processed to peripheral blood mononuclear cells at the conclusion of the run-in phase and after 16 weeks of diet exposure. All aliquots were prepared and stored at $-80^{\circ} \mathrm{C}$ until analysis, with transport on dry ice to prevent thawing. Laboratory staff masked to treatment groups performed assays. Targeted profiling of oxylipins in the free pool of serum and the total (free plus esterified) pool of plasma was performed using ultra performance liquid chromatography, tandem mass spectrometry in the Laboratory of Clinical Investigation, National Institute on Aging. Free serum oxylipins were assayed as previously described. ${ }^{54}$ For total oxylipins, plasma lipids were hydrolyzed with sodium hydroxide using methods adapted from Ostermann and colleagues, ${ }^{55}$ extracted with Oasis-HLE solid phase extraction cartridges, and profiled by the same tandem mass spectrometry methods as used for serum free acids. ${ }^{54}$ Enzyme linked immunosorbent assays (ELISAs) for prostaglandin E2, leukotriene B4, cysteinyl leukotrienes, and calcitonin gene related peptide were performed at the University of North Carolina at Chapel Hill, Cytokine Analysis Core according to manufacturer instructions. Precursor fatty acids in erythrocytes and immune cells (peripheral blood mononuclear cells) were analyzed by gas chromatography with flame ionization detector, as previously described, ${ }^{6}$ in the Laboratory of Membrane Biochemistry and Biophysics in the Intramural Program of the National Institute on Alcohol Abuse and Alcoholism.

\section{Statistical analysis}

The 17-HDHA, HIT-6, and headache hours per day endpoints were prespecified as specific aims one and two in our published protocol, ${ }^{45}$ with 17-HDHA and HIT- 6 as the primary biochemical and clinical outcomes. Estimations based on our previous trial in a chronic daily headache population ${ }^{6}$ predicted that a sample of 153 patients (51 per group) would provide more than $90 \%$ power to detect a mean difference of 0.7 (standard deviation 0.7 ) $\log \mathrm{ng} / \mathrm{mL}$ in plasma 17-HDHA between the H3-L6 group and the control group at a two sided $\alpha$ of 0.0167 (overall $\alpha$ of 0.05 and Bonferroni adjusted $\alpha$ of 0.0167 for between group comparisons). With an anticipated rate of discontinuation of $15 \%, 182$ participants were planned for randomization. At this $\alpha$ and sample size, the expected power to detect differences in 17-HDHA between $\mathrm{H} 3$ and control groups and between H3-L6 and $\mathrm{H} 3$ groups ranged from $71 \%$ to $95 \%$. The expected power to detect between group differences of three to five points in HIT-6 ranged from 54\% to 99\%. Analyses were conducted in Stata version $16 .^{56}$

The prespecified primary analysis for 17-HDHA (natural log transformed) and HIT-6 was an analysis of covariance, with the week 16 values as dependent variables and indicator variables included for the diet group and adjusted for recruitment site and baseline values. Longitudinal analyses using generalized estimating equation models were prespecified for assessment of between group differences in variables from the headache diary (eg, headache hours per day and headache days per month). These models included continuous group by time interactions and were adjusted for recruitment site and baseline value of the respective outcome (defined as the mean of the 28 days before randomization). Headache 
days per month was analyzed with population averaged (generalized estimating equation) Poisson regression with autoregressive structure for within person correlation. Headache hours per day and the number of drug use instances per day were analyzed with population averaged negative binomial regression models to account for overdispersion in the values of the daily data. ${ }^{57}$ Analyses of between group differences in 17-HDHA, HIT-6, and headache frequency included all randomized participants unless otherwise specified. Missing data were imputed by using multiple imputation procedures (within group chained equations using a predictive mean matching algorithm generating 30 imputations per missing value). Variables included in the imputation model were personal characteristics, headache outcomes, sleep quality, stress, overall health, drug use (including botulinum toxin), body weight and height, expectation of benefit, recruitment site, and selected oxylipins. We ran analysis models for each of the 30 imputed datasets, and the results were combined using Rubin's rules.

We conducted several post hoc heterogeneity and sensitivity analyses. Heterogeneity analyses to estimate differences in diet effects on headache related outcomes according to participants' baseline episodic versus chronic migraine and continuous body mass index were conducted by adding group interaction terms and testing that the interaction coefficients equal zero. Associations between baseline credibility scores and headache related outcomes at end of study were calculated using linear regressions. We calculated between group differences in the use of preventive drugs by using $\chi^{2}$ tests, and sensitivity analyses were conducted adjusting for drug categories with relevant between group differences. To further explore diet effects on drug use, we calculated the number of participants meeting criteria for acute drug overuse at end of study and used logistic regression to compare between group odds ratios of drug overuse. This analysis was restricted to participants who filled out the drug use section of the electronic headache diary on at least 10 days in the last month of the intervention.

Statistical analyses of blood fatty acids and oxylipins (other than the primary biochemical outcome) included only participants with complete data at baseline and week 16. For participants who dropped out before week 16, data from the last follow-up visit completed (week 10 or week 4) were substituted whenever available. Analysis of covariance (with variable transformations, if necessary) was used to assess between group differences at the final visit.

A secondary prespecified aim in our published protocol $^{45}$ was to explore the hypotheses that diet induced increases in blood n-3 EPA and DHA fatty acids (and their antinociceptive oxylipin derivatives) would be positively associated with improvement in HIT-6 score and mean headache hours per day; and that diet induced reductions in blood n-6 linoleic acid and arachidonic acid (and their pronociceptive oxylipin derivatives) would be positively associated with improvement in HIT-6 score and mean headache hours per day. The independent variables in this analysis were the four targeted erythrocyte fatty acids and their measured oxylipin derivatives in plasma for which diet induced changes were evident with a Bonferroni adjusted $\alpha$ of 0.0167 (4-HDHA, 10-HDHA, 14-HDHA, 17-HDHA, and 5-hydroxyeicosatetraenoic acid (5-HETE)). Only participants with data available for both blood measures and endpoints were included in this analysis. These linear regression models were adjusted for several baseline variables: age, body mass index, headache days per month, HIT-6, chronic versus episodic migraine, and sex.

\section{Patient and public involvement}

Patients were not involved in the design, implementation, or reporting of the study.

\section{Results}

Overall, 182 participants were randomized to one of three diets: H3-L6 ( $n=61), H 3(n=61)$, or control $(\mathrm{n}=60) ; 154$ of the 182 randomized participants $(85 \%)$ completed $\geq 10$ weeks of intervention, and 141 (77\%) completed the 16 week intervention (fig 2). Personal and baseline characteristics were comparable between groups, with mean age of 38.3 years, body mass index of 29.4, and 161 (88\%) women in the total sample (table 1). Baseline mean HIT-6 score was 62.7 (standard deviation 5.3), indicating a severe impact of headaches on quality of life. ${ }^{50}$ At study entry, participants averaged 16.3 headache days per month and 5.4 headache hours per day, despite reporting an average of 4.3 headache related drugs per person; 122 participants (67\%) had chronic migraine and 102 (56\%) met criteria for acute drug overuse. After the first dietitian counseling session but before the provision of study foods designed to provide equivalent credibility across the groups, the mean credibility scores for the H3 (35.9) and the H3-L6 (35.2) groups were slightly higher than the control group (32.2).

\section{Primary endpoints}

In intention-to-treat analyses $(n=182$; table 2$)$, the H3 and H3-L6 diets increased circulating 17-HDHA (log $\mathrm{ng} / \mathrm{mL}$ ) compared with the control group. The baselineadjusted mean differences were 0.7 (95\% confidence interval 0.4 to $1.1, \mathrm{P}<0.001)$ and 0.6 ( 0.2 to 0.9 , $\mathrm{P}=0.001)$, respectively. The observed improvement in HIT-6 scores in the H3 and H3-L6 groups was clinically relevant, ${ }^{52}$ but not statistically significant $(-1.5,-4.2$ to 1.2 , and $-1.6,-4.2$ to 1.0 , respectively).

\section{Headache frequency and severity}

The mean baseline-adjusted headache hours per day at end of study were 4.9 (95\% confidence interval 4.2 to 5.6) in the control group, 3.6 (3.1 to 4.1) in the $\mathrm{H} 3$ group, and 3.2 (2.8 to 3.7) in the H3-L6 group (table 2). Compared with the control group, the H3 and the H3-L6 groups significantly reduced the number of total headache hours per day $(-1.3,-2.1$ to -0.5 , and $-1.7,-2.5$ to -0.9 , respectively) and moderate to 


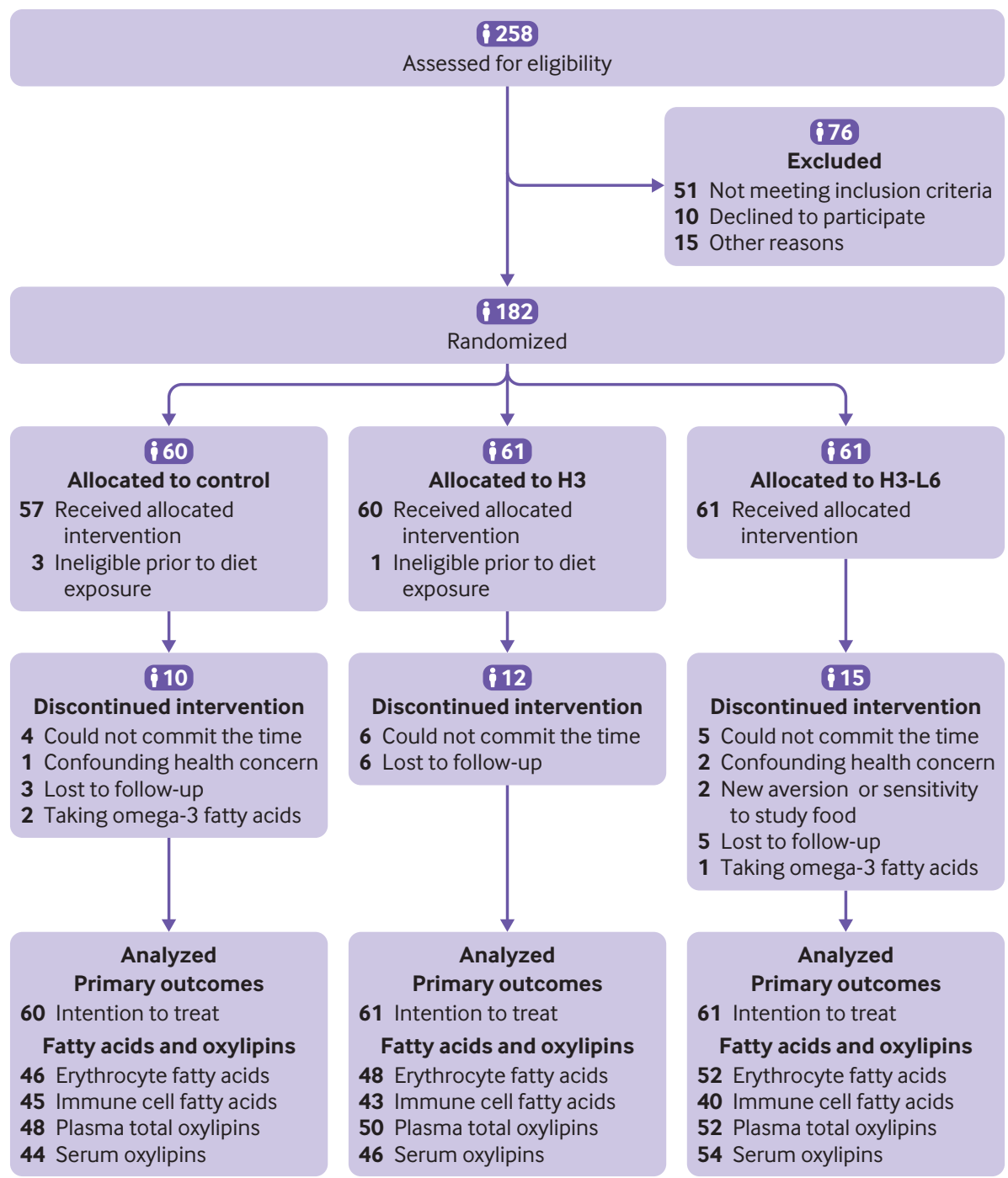

Fig 2 | CONSORT (consolidated standards of reporting trials) diagram

severe headache hours per day $(-0.7,-1.1$ to -0.3 , and $-0.8,-1.2$ to -0.4 , respectively; $\mathrm{P} \leq 0.001$ for all comparisons with control group, including group by time interactions). The H3-L6 group had four fewer headache days per month compared with the control group $(-4.0,-5.2$ to $-2.7, \mathrm{P}<0.001)$ and two fewer headache days per month compared with the $\mathrm{H} 3$ group $(-2.0,-3.2$ to $-0.8, \mathrm{P}=0.001)$.

\section{Heterogeneity and sensitivity analyses}

Table S3 and figure S1 give results of heterogeneity analyses for the effects of the interventions on 17 HDHA, HIT-6, headache hours per day, and headache days per month. Overall, the effects of the interventions were not statistically different for participants with episodic migraine compared with those with chronic migraine. Additionally, no evidence of statistical heterogeneity in treatment effects was found across body mass index values for the 17-HDHA and HIT6 outcomes, but heterogeneity was present for the headache hours per day and headache days per month endpoints. While headache frequency tended to be higher with higher body mass index in the control group, patients in the H3-L6 group with higher body mass index values experienced a larger reduction in headache (fig S1). The effect of the $\mathrm{H} 3$ diet on headache frequency was relatively constant across body mass index values.

In an analysis exploring the association between credibility scores at baseline and headache endpoints at end of study, credibility scores were not predictive of later improvement. For example, the coefficients for a one unit increase in credibility score were 0.02 (95\% confidence interval -0.11 to 0.16 ) and -0.06 ( -0.18 to 0.06$)$ for HIT6 and headache days, respectively (table S4).

Figure $\mathrm{S} 2$ shows the use of preventive drugs reported for headache treatment (including antidepressants, anticonvulsants, $\beta$ blockers, muscle relaxants) at baseline and follow-up. One hundred and three participants $(57 \%)$ reported using any preventive drugs at baseline and few changes were reported throughout the study. At baseline, the number of participants using any preventive drugs was 33 in the control group, 32 in the $\mathrm{H} 3$ group, and 38 in the H3L6 group. Overall, little change was found between the baseline and final numbers. The use of most categories 


\begin{tabular}{|c|c|c|c|c|}
\hline Characteristics & Control $(n=60)$ & H3 $(n=61)$ & H3-L6 $(n=61)$ & Total $(n=182)$ \\
\hline \multicolumn{5}{|l|}{ Personal characteristics } \\
\hline Age (years), mean (SD) & $36.9(12.5)$ & $38.8(11.9)$ & $39.4(11.7)$ & $38.3(12.0)$ \\
\hline Body mass index, mean (SD) & $29.3(7.3)$ & $29.1(8.4)$ & $29.8(11.3)$ & $29.4(9.1)$ \\
\hline Female participants & $52(87)$ & $52(85)$ & $57(93)$ & $161(88)$ \\
\hline \multicolumn{5}{|l|}{ Race } \\
\hline Black & $11(18)$ & $8(13)$ & $14(23)$ & $33(18)$ \\
\hline White & $47(78)$ & $45(74)$ & $46(75)$ & $138(76)$ \\
\hline Other & $2(3)$ & $8(13)$ & $1(2)$ & $11(6)$ \\
\hline Non-Hispanic & $58(97)$ & $58(95)$ & $58(95)$ & $174(96)$ \\
\hline \multicolumn{5}{|l|}{ Relationship status } \\
\hline Single & $13(22)$ & $13(21)$ & $14(23)$ & $40(22)$ \\
\hline Partnered & $42(70)$ & $39(64)$ & $38(62)$ & $119(65)$ \\
\hline Divorced or separated & $4(7)$ & $8(13)$ & $8(13)$ & $20(11)$ \\
\hline \multicolumn{5}{|l|}{ Highest level of education completed } \\
\hline High school diploma, general education development, or lower & $4(7)$ & $3(5)$ & $6(10)$ & $13(7)$ \\
\hline Some college or bachelor's degree & $37(62)$ & $36(59)$ & $36(59)$ & $109(60)$ \\
\hline Postgraduate & $18(30)$ & $22(36)$ & $18(30)$ & $58(32)$ \\
\hline \multicolumn{5}{|l|}{ Annual household income (\$) } \\
\hline$<20000$ & $14(23)$ & $8(13)$ & $5(8)$ & $27(15)$ \\
\hline $21000-40000$ & $14(23)$ & $17(28)$ & $11(18)$ & $42(23)$ \\
\hline $41000-60000$ & $7(12)$ & $9(15)$ & $17(28)$ & $33(18)$ \\
\hline $61000-80000$ & $8(13)$ & $6(10)$ & $7(11)$ & $21(12)$ \\
\hline 180000 & $16(27)$ & $20(33)$ & $17(28)$ & $53(29)$ \\
\hline No of people supported by household income, mean (SD) & $2.1(1.2)$ & $2.6(1.5)$ & $2.9(1.9)$ & $2.5(1.6)$ \\
\hline \multicolumn{5}{|l|}{ Clinical characteristics } \\
\hline HIT-6 score, mean (SD) & $62.3(5.7)$ & $62.7(5.6)$ & $63.2(4.7)$ & $62.7(5.3)$ \\
\hline Headache hours per day, mean (SD) & $5.1(4.1)$ & $5.7(4.3)$ & $5.5(4.0)$ & $5.4(4.1)$ \\
\hline Moderate to severe headache hours per day, mean (SD) & $2.0(2.1)$ & $2.1(2.1)$ & $2.0(1.8)$ & $2.0(2.0)$ \\
\hline Headache days per month, mean (SD) & $16.3(6.3)$ & $16.5(6.2)$ & $16.0(6.4)$ & $16.3(6.3)$ \\
\hline Migraine days per month, mean (SD) & $10.6(3.9)$ & $10.2(4.1)$ & $10.9(4.1)$ & $10.6(4.0)$ \\
\hline Chronic migraine $^{\star}$ & $42(70)$ & $42(69)$ & $38(62)$ & $122(67)$ \\
\hline Migraine with aura & $18(30)$ & $14(23)$ & $19(31)$ & $51(28)$ \\
\hline Age at first onset of headaches, mean (SD) & $17.0(9.5)$ & $16.9(11.7)$ & $17.0(8.3)$ & $17.0(9.9)$ \\
\hline Age at most recent onset of headaches, mean (SD) & $25.5(10.5)$ & $26.2(13.7)$ & $28.2(10.8)$ & $26.5(11.7)$ \\
\hline Acute pain drug overuset & $36(60)$ & $31(51)$ & $35(57)$ & $102(56)$ \\
\hline Total number of headache related pain drugs, mean (SD) $\ddagger$ & $4.2(3.0)$ & $4.2(3.2)$ & $4.5(3.4)$ & $4.3(3.2)$ \\
\hline Preventive, mean (SD) & $0.9(1.1)$ & $1.1(1.4)$ & $1.3(1.5)$ & $1.1(1.3)$ \\
\hline Acute, mean (SD) & $2.0(1.4)$ & $1.9(1.2)$ & $2.0(1.4)$ & $2.0(1.3)$ \\
\hline Adjunctive, mean (SD) & $1.2(1.6)$ & $1.2(1.6)$ & $1.1(1.4)$ & $1.2(1.6)$ \\
\hline Credibility rating for assigned intervention (0-45), mean (SD)§ & $32.2(7.7)$ & $35.9(7.4)$ & $35.2(8.3)$ & $34.5(7.9)$ \\
\hline \multicolumn{5}{|c|}{ 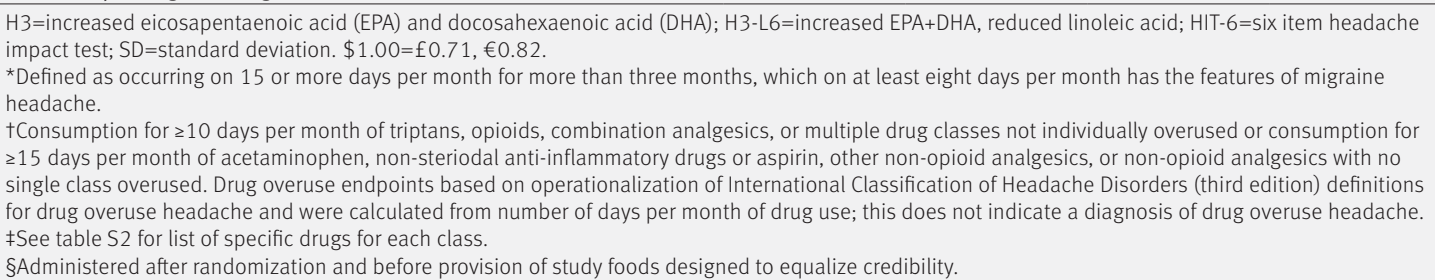 } \\
\hline
\end{tabular}

of preventive drugs was similar across diet groups, except anticonvulsants. At baseline, the number of participants using anticonvulsants was six in the control group, 19 in the H3 group, and 23 in the H3L6 group. A sensitivity analysis adjusting the table 2 models for confounding by baseline anticonvulsant use did not materially change the findings (table S5).

\section{Acute headache related drugs}

In intention-to-treat analysis of the number of instances per day that participants used acute headache related drugs (table 2), the $\mathrm{H} 3$ diet decreased total instances of acute drug use (including NSAIDs, acetaminophen, triptans) compared with the control diet $(-0.4,95 \%$ confidence interval -0.7 to $-0.1, \mathrm{P}=0.01)$; the H3-L6 diet did not alter total acute pain drug use $(-0.04,-0.4$ to $0.3, \mathrm{P}=0.83$ ). Both diets decreased the use of NSAIDs or aspirin compared with the control diet (H3-L6 diet: $-0.2,-0.4$ to $-0.1, \mathrm{P}=0.01$; H3 diet: $-0.1,-0.3$ to 0.1 , $\mathrm{P}=0.20$ ), and the difference between the effect of the $\mathrm{H} 3$ L6 and $\mathrm{H} 3$ diets was relatively small $(-0.1,-0.3$ to 0.04 , $\mathrm{P}=0.13$ ). Both intervention diets did not decrease the instances of use of migraine abortive drugs (triptans).

Among the 143 participants who filled out the drug section of the electronic headache diary in the last month of the study, the number of participants classified with acute drug overuse was 24 of $48(50 \%)$ in the control group, 26 of 47 (55\%) in the H3 group, and 19 of 48 (40\%) in the H3-L6 group (table S6). In logistic regression adjusted for the baseline number of days of acute drug 


\begin{tabular}{|c|c|c|c|}
\hline Endpoints & Control $(n=60)$ & H3 $(n=61)$ & H3-L6 ( $n=61)$ \\
\hline \multicolumn{4}{|l|}{ Primary endpoints $t$} \\
\hline \multicolumn{4}{|l|}{ 17-HDHA in plasma $(\log \mathrm{ng} / \mathrm{mL})$} \\
\hline Estimate $(95 \% \mathrm{Cl})$ & $0.7(0.4$ to 0.9$)$ & $1.4(1.2$ to 1.6$)$ & $1.2(1.0$ to 1.5$)$ \\
\hline Difference $v$ control $(95 \%$ Cl) & - & $0.7(0.4$ to 1.1$)$ & $0.6(0.2$ to 0.9$)$ \\
\hline$P$ value & - & $<0.001$ & 0.001 \\
\hline Cohen's d & - & 0.83 & 0.64 \\
\hline \multicolumn{4}{|l|}{ HIT-6 score } \\
\hline Estimate $(95 \% \mathrm{Cl})$ & $59.9(58.0$ to 61.8$)$ & $58.4(56.5$ to 60.3$)$ & $58.3(56.5$ to 60.2$)$ \\
\hline Difference $v$ control $(95 \% \mathrm{Cl})$ & - & $-1.5(-4.2$ to 1.2$)$ & $-1.6(-4.2$ to 1.0$)$ \\
\hline Pvalue & - & 0.27 & 0.23 \\
\hline Cohen's d & - & -0.21 & -0.22 \\
\hline \multicolumn{4}{|l|}{ Secondary endpoints $¥$} \\
\hline \multicolumn{4}{|l|}{ Headache hours per day } \\
\hline Estimate $(95 \% \mathrm{Cl})$ & $4.9(4.2$ to 5.6$)$ & $3.6(3.1$ to 4.1$)$ & $3.2(2.8$ to 3.7$)$ \\
\hline Difference $v$ control $(95 \% \mathrm{Cl})$ & - & $-1.3(-2.1$ to -0.5$)$ & $-1.7(-2.5$ to -0.9$)$ \\
\hline Pvalue & - & 0.001 & $<0.001$ \\
\hline Cohen's d & - & -0.56 & -0.71 \\
\hline \multicolumn{4}{|c|}{ Moderate to severe headache hours per day } \\
\hline Estimate $(95 \% \mathrm{Cl})$ & 2.1 (1.7 to 2.5$)$ & $1.4(1.1$ to 1.6$)$ & $1.3(1.0$ to 1.6$)$ \\
\hline Difference $v$ control $(95 \% \mathrm{Cl})$ & - & $-0.7(-1.1$ to -0.3$)$ & $-0.8(-1.2$ to -0.4$)$ \\
\hline Pvalue & - & $<0.001$ & $<0.001$ \\
\hline Cohen's d & - & -0.58 & -0.62 \\
\hline \multicolumn{4}{|l|}{ Headache days per month } \\
\hline Estimate $(95 \% \mathrm{Cl})$ & $13.2(12.2$ to 14.2$)$ & $11.2(10.4$ to 12.1$)$ & $9.2(8.4$ to 10.0$)$ \\
\hline Difference $(95 \% \mathrm{Cl})$ & - & $-2.0(-3.3$ to -0.7$)$ & $-4.0(-5.2$ to -2.7$)$ \\
\hline Pvalue & - & 0.003 & $<0.001$ \\
\hline Cohen's d & - & -0.55 & -1.13 \\
\hline \multicolumn{4}{|c|}{ Acute pain drugs, instances per day§ } \\
\hline \multicolumn{4}{|c|}{ Total } \\
\hline Estimate $(95 \% \mathrm{Cl})$ & $1.3(1.0$ to 1.6$)$ & $0.9(0.7$ to 1.1$)$ & $1.2(1.0$ to 1.5$)$ \\
\hline Difference $v$ control $(95 \% \mathrm{Cl})$ & - & $-0.4(-0.7$ to -0.1$)$ & $-0.04(-0.4$ to 0.3$)$ \\
\hline P value & - & 0.01 & 0.83 \\
\hline Cohen's d & - & -0.37 & -0.03 \\
\hline \multicolumn{4}{|l|}{ NSAIDs or aspirin } \\
\hline Estimate $(95 \% \mathrm{Cl})$ & $0.6(0.4$ to 0.8$)$ & $0.5(0.3$ to 0.6$)$ & $0.4(0.3$ to 0.4$)$ \\
\hline Difference $v$ control $(95 \% \mathrm{Cl})$ & - & $-0.1(-0.3$ to 0.1$)$ & $-0.2(-0.4$ to -0.1$)$ \\
\hline Pvalue & - & 0.20 & 0.01 \\
\hline Cohen's d & - & -0.20 & -0.43 \\
\hline \multicolumn{4}{|l|}{ Triptans } \\
\hline Estimate $(95 \% \mathrm{Cl})$ & $0.3(0.2$ to 0.3$)$ & $0.2(0.1$ to 0.3$)$ & $0.2(0.2$ to 0.3$)$ \\
\hline Difference $(95 \% \mathrm{Cl})$ & - & $-0.1(-0.2$ to 0.02$)$ & $-0.02(-0.1$ to 0.1$)$ \\
\hline Pvalue & - & 0.13 & 0.63 \\
\hline Cohen's d & - & -0.24 & -0.08 \\
\hline \multicolumn{4}{|l|}{ Other } \\
\hline Estimate $(95 \% \mathrm{Cl})$ & $0.4(0.3$ to 0.6$)$ & $0.3(0.2$ to 0.3$)$ & $0.6(0.4$ to 0.7$)$ \\
\hline Difference $v$ control $(95 \% \mathrm{Cl})$ & - & $-0.2(-0.3$ to -0.02$)$ & $0.1(-0.04$ to 0.3$)$ \\
\hline Pvalue & - & 0.02 & 0.14 \\
\hline Cohen's d & - & -0.37 & 0.23 \\
\hline \multicolumn{4}{|c|}{ 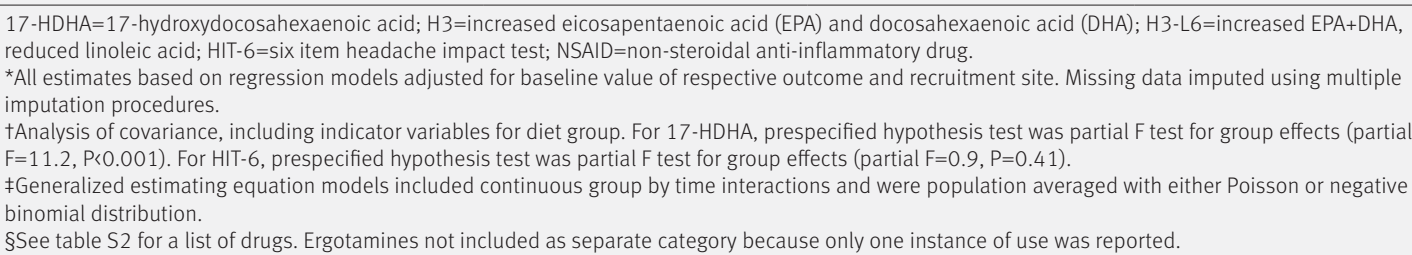 } \\
\hline
\end{tabular}

use, the odds ratio for overall acute drug overuse was 1.74 for the $\mathrm{H} 3$ group versus the control group (95\% confidence interval 0.59 to $5.10, \mathrm{P}=0.31$ ) and 0.45 for the H3-L6 group versus the control group ( 0.15 to 1.40 , $\mathrm{P}=0.17$ ). The individual categories of overuse showed similar trends, with the H3-L6 group having fewer participants with overuse, although the differences were small in absolute numbers and had wide confidence intervals. NSAID overuse ( $\geq 15$ days per month) was lower in the H3-L6 group (2 of 48 participants) compared with the control group (7 of 48 participants), with an adjusted odds ratio of 0.10 (95\% confidence interval 0.01 to 0.76 ; $\mathrm{P}=0.03)$. Overall, no indication was found that the $\mathrm{H} 3$ diet reduced acute drug overuse in this sample.

\section{Adverse events}

We did not observe between group differences in adverse events (table S7). 


\section{Study diets}

Table S8 shows targets and achieved intakes of dietary fatty acids, estimated by 24 hour recalls. The H3 intervention was designed to increase n-3 EPA+DHA as a controlled variable to $1.5 \mathrm{~g} /$ day while holding $\mathrm{n}-6$ linoleic acid and all other nutrients constant; the H3L6 diet was designed to achieve the same EPA+DHA targets and to also decrease n- 6 linoleic acid from around $7 \%$ of energy to $1.8 \%$ of energy. The $\mathrm{H} 3$ and the H3-L6 groups both achieved >30-fold increases in median intakes of n-3 EPA+DHA compared with baseline and the control group, but both groups fell slightly short of the targeted goal. The H3-L6 group lowered dietary n-6 linoleic acid to a median of 3.2\% of energy (interquartile range 2.7-4.4) but did not reach the target of $1.8 \%$. Neither the H3 nor the H3-L6 diet caused meaningful changes in body weight (table S9).

\section{Oxylipin precursors in circulation}

The H3 and H3-L6 interventions increased EPA+DHA in erythrocytes and immune cells $(\mathrm{P}<0.001$; fig 3 and tables S10-S11), with EPA+DHA medians for each group 46-95\% higher than the control diet. Both interventions also significantly decreased n-6 arachidonic acid and its elongation product docosatetraenoic acid in erythrocytes compared with the control diet. The H3-L6 intervention decreased the concentration of linoleic acid and its elongation product eicosadienoic acid in erythrocytes compared with the $\mathrm{H} 3$ and control diets, and decreased the linoleic acid content of immune cells compared with the $\mathrm{H} 3$ diet.

\section{Antinociceptive and pronociceptive oxylipins}

The H3-L6 and H3 interventions increased 4-HDHA, 10-HDHA, 14-HDHA, and 17-HDHA derivatives of DHA in the total (free plus esterified) pool of plasma and the free pool of serum (fig 4 and tables S12-S13). Compared with the control diet, the $\mathrm{H} 3$ diet decreased 5-HETE (a 5-lipoxygenase derivative of arachidonic acid; $-3.2 \mathrm{ng} / \mathrm{mL}, 95 \%$ confidence interval -5.6 to $-0.9)$ and decreased 8-HETE and 15-HETE $(-1.5 \mathrm{ng} /$ $\mathrm{mL},-3.0$ to 0.02 , and $-2.9 \mathrm{ng} / \mathrm{mL},-5.8$ to -0.02 , respectively) in the total pool. The H3-L6 diet had no effect on HETEs. Additionally, the H3-L6 diet did not decrease any oxidized derivatives of linoleic acid in the total or the free pool. Both diets did not alter classic arachidonic acid derived oxylipin mediators of pain and inflammation (prostaglandin E2, leukotriene B4, or cysteinyl leukotrienes), or the migraine related neuropeptide calcitonin gene related peptide measured by ELISA (fig 4 and table S12).

\section{Associations between biochemical measures and headache endpoints}

Figure 5 shows plots for the associations of headache hours per day with targeted erythrocyte fatty acids and plasma oxylipins at end of study. Increases in EPA and DHA and decreases in linoleic acid (as percentage of total fatty acids) were associated with lower headache hours per day $(\mathrm{P}=0.001,0.001$, and 0.03 , respectively).
Increases in DHA derived oxylipins (4-HDHA, 10HDHA, 14-HDHA, and 17-HDHA) were also associated with lower headache hours per day $(\mathrm{P}<0.001,<0.001$, 0.02 , and $<0.001$, respectively). No association was found between 5-HETE and headache hours per day. Figure S2 shows plots with HIT-6 as the dependent outcome. While directionally consistent with those in figure 5, the associations of HIT-6 and circulating fatty acids and oxylipins were mostly weak or non-existent.

\section{Discussion}

The overarching hypothesis underlying this randomized trial was that increasing dietary n-3 EPA+DHA (with or without concurrent reduction in n-6 linoleic acid) would alter nociceptive oxylipins in a manner that would decrease headache frequency and severity. Additionally, any such improvement would be reflected in a monthly recall measure designed to capture headache impact on various aspects of daily life (HIT6). While the biochemical results are consistent with our mechanistic model, the clinical results were mixed and require a more nuanced interpretation. After 16 weeks, the improvement in HIT-6 in the H3 and H3L6 diets was consistent with the minimally important difference of 1.5 points, ${ }^{52}$ but it was not statistically significant compared with the control diet. However, the $\mathrm{H} 3$ and H3-L6 diets produced robust reductions in the frequency and severity of headaches (as recorded in an electronic headache diary). While the items in the HIT-6 might better reflect patients' own evaluation of how headaches affect their life, the electronic headache diary provides a more specific evaluation of physical pain by prompting participants to record details of headache presence, duration, and severity on a daily basis with little potential for recall bias.

Headache frequency and severity endpoints from the electronic headache diary were prespecified secondary outcomes in our published protocol. ${ }^{45}$ The H3 and H3-L6 diets decreased the number of headache hours per day and moderate to severe headache hours per day compared with the control diet. Improvements in headache days per month in the H3-L6 group were greater than those in the H3 group, suggesting additional benefit from lowering dietary n-6 linoleic acid. The observed reductions in headache frequency endpoints in the H3-L6 group (Cohen's d ranging from -0.62 to -1.13 ) were comparable to those observed in a previous, smaller randomized trial testing a similar intervention in 67 patients with chronic daily headache. ${ }^{6}$ The mean reductions of 1.7 headache hours per day and 4.0 headache days per month in the H3-L6 group versus the control group are comparable to those recently reported for botulinum toxin injections ( -2.4 migraine days per month), ${ }^{58}$ and monoclonal antibodies targeting calcitonin gene related peptide $(-1.8$ to -2.1 days per month with a composite headache endpoint). ${ }^{59}$ These findings suggest that the intervention might be an efficacious adjunct approach for managing headache.

At study entry, $122(67 \%)$ participants met the criteria for chronic migraine, which is a notoriously 

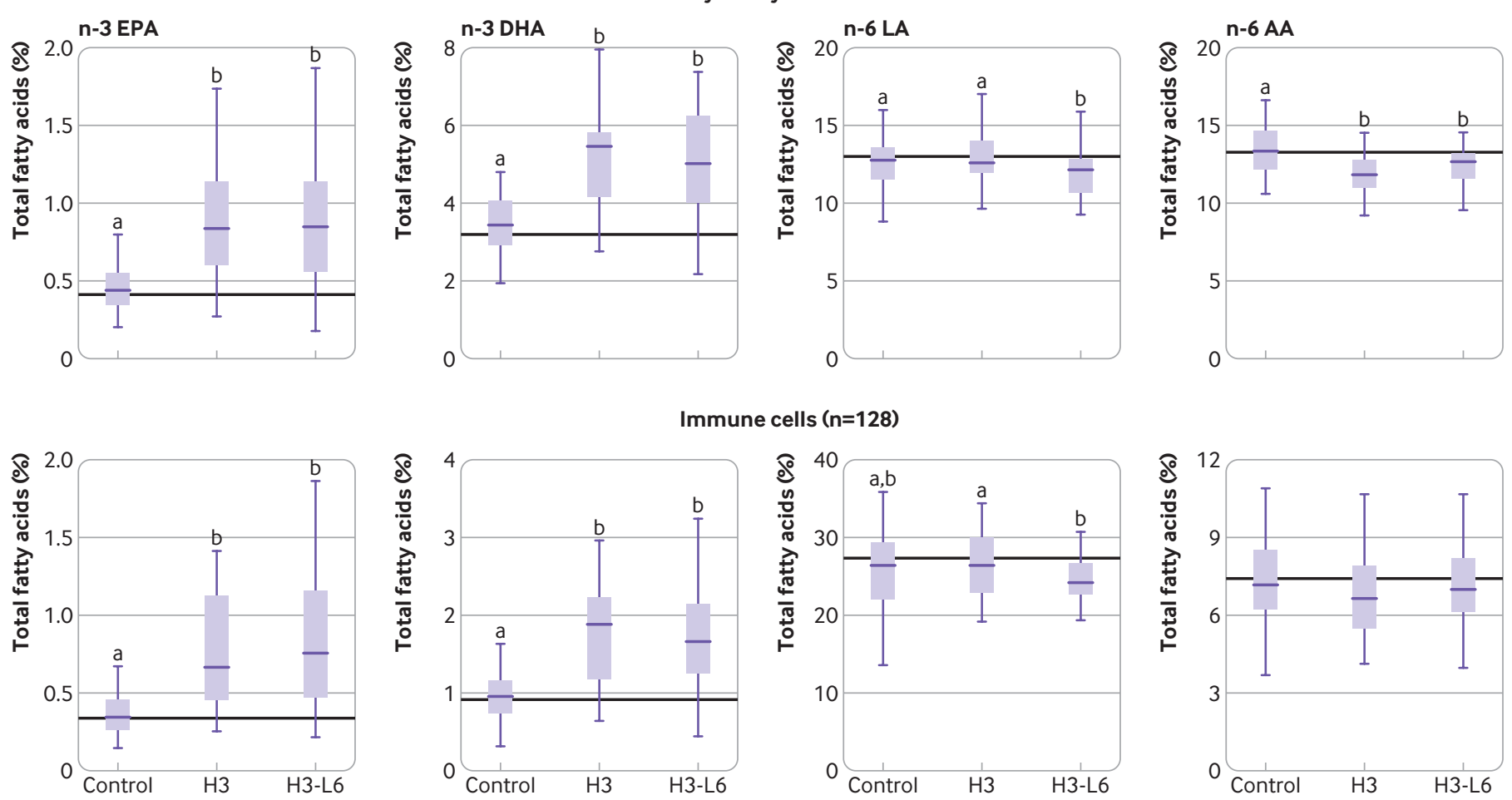

Fig 3 | Effect of study diets on precursor fatty acids. Distributions of the final measure of three pools of precursor fatty acids: erythrocytes and immune cells. Boxes represent median and interquartile range. Outliers beyond whiskers are not shown. For reference, baseline median is represented by black line. To assess between group differences at final visit, an analysis of covariance was used, controlling for recruitment site. Different letters indicate $P$ values $<0.05$ between groups. For details on these and other fatty acids, see tables S10-S11. AA=arachidonic acid; DHA=docosahexaenoic acid; EPA=eicosapentaenoic acid; H3=increased eicosapentaenoic acid (EPA) and docosahexaenoic acid (DHA); H3$\mathrm{L} 6=$ increased $\mathrm{EPA}+\mathrm{DHA}$, reduced linoleic acid; $\mathrm{LA}=$ linoleic acid

difficult condition to treat. $^{60-62}$ In a post hoc heterogeneity analysis by baseline migraine diagnosis, the H3 and H3-L6 diets seemed effective in reducing headache days per month among participants with chronic migraine ( -1.8 and -4.4 days, respectively), and among participants with episodic migraine $(-2.7$ and -3.6 days, respectively). The lack of heterogeneity in treatment effects also applied to HIT-6 and 17-HDHA. This finding suggests that the interventions are likely to have similar efficacy in episodic migraine and chronic migraine populations, although larger populations are needed to clarify this suggestion. In similar post hoc analyses, we observed that participants with higher baseline body mass index experienced a larger reduction in headaches with the H3-L6 diet (fig S1). This might be a relevant finding because people with higher body mass index tend to have more headaches and are at increased risk of migraine (episodic and chronic). ${ }^{63}{ }^{64}$ Because our three diets were designed to equalize intake of non-controlled foods and to keep participants' weight stable throughout the study period, it is unlikely that changes in body composition or in the intake of non-controlled foods explain these findings.

Drug sparing effects of $\mathrm{H} 3$ and H3-L6 interventions Pain relieving drugs are well known to have side effects and drug overuse is considered a major problem in patients with migraine. ${ }^{65}$ The observation that $\mathrm{H} 3$ and H3-L6 interventions decreased headaches while concurrently decreasing the use of total acute headache related drugs or NSAIDS specifically is therefore a potentially important finding. Firstly, this observation supports the proposed biochemical mechanisms (fig 1) because NSAIDs work by inhibiting conversion of n- 6 arachidonic acid to 2 series prostaglandins, a class of oxylipins closely linked to pain; this is one of the biochemical pathways we targeted with the $\mathrm{H} 3$ and H3-L6 diets (eg, increasing intake of EPA+DHA lowers concentration of arachidonic acid in membranes, ${ }^{66}$ thus decreasing substrate for conversion to 2 series prostaglandins). Secondly, the finding suggests that the use of mechanism based dietary alterations to regulate pain upstream of where most current drugs would act could potentially reduce drug (over)use and associated risks.

\section{Investigating mechanisms of pain reduction}

At baseline, participants had relatively high amounts of $n-6$ linoleic acid and arachidonic acid and low n-3 EPA and DHA in their erythrocytes and immune cells, consistent with modern industrialized diets. Our mechanistic model (fig 1) suggests that this intake pattern is conducive to a pronociceptive state. The H3 and H3-L6 diets produced biochemical changes consistent with a decreased nociception, such as 
Total $n-3$ oxylipins in plasma $(n=150)$
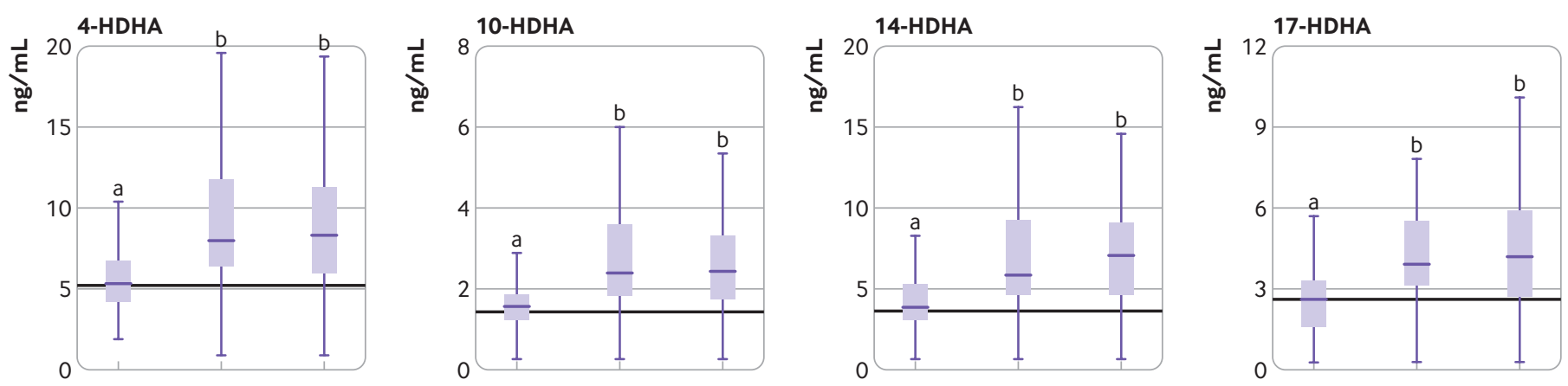

Total $n-6$ oxylipins in plasma $(n=150)$
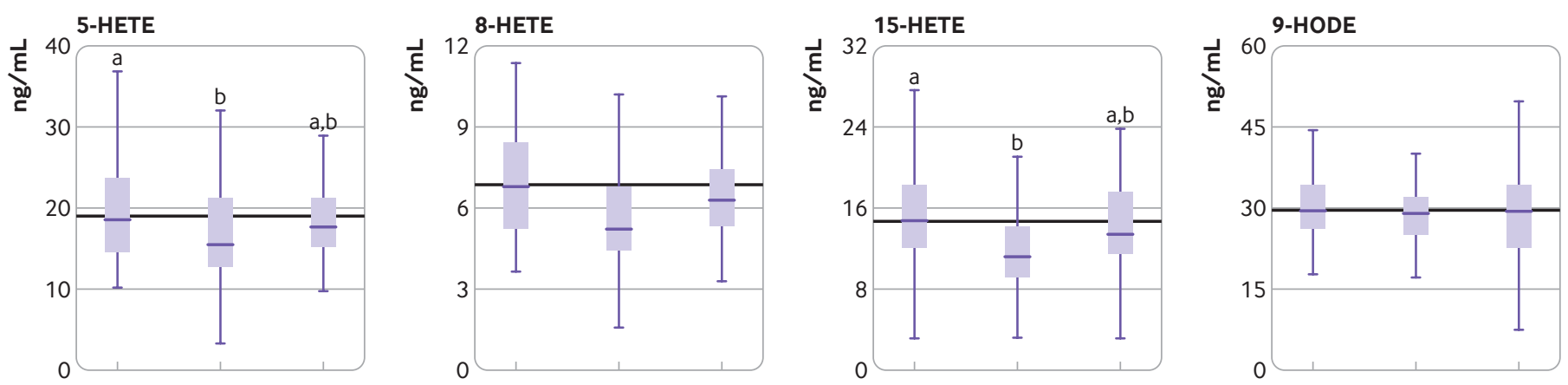

Plasma oxylipins and CGRP measured by ELISA
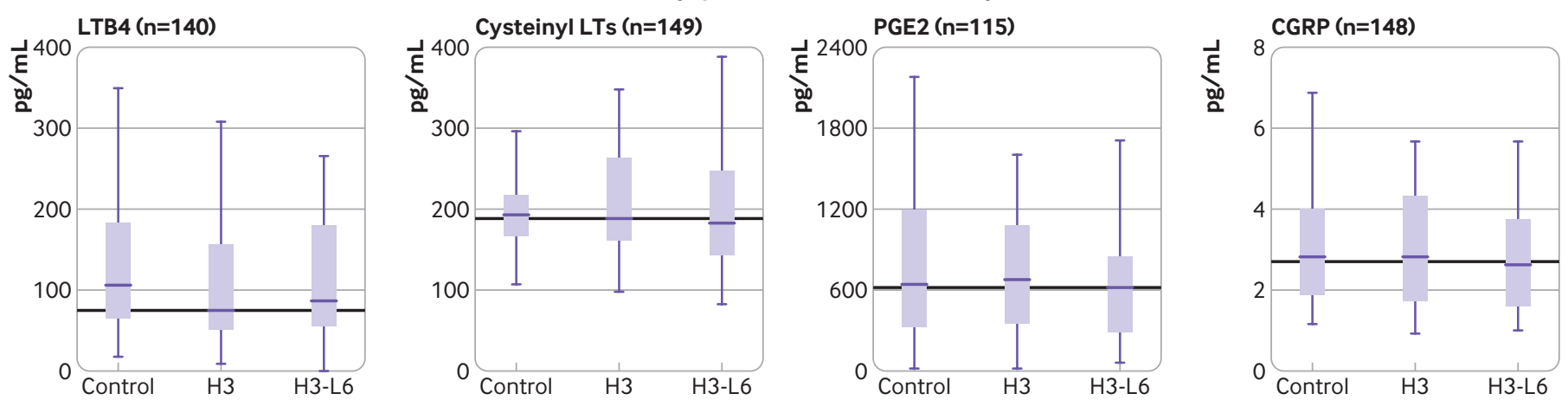

Fig 4 | Effect of study diets on oxylipins and CGRP. Distributions of final measure of oxylipins and CGRP in plasma. Boxes represent median and interquartile range. Outliers beyond whiskers are not shown. For reference, baseline median is represented by black line. To assess between group differences at final visit, an analysis of covariance was used, controlling for recruitment site. Different letters indicate $P$ values $<0.05$ between groups. For details on these and other oxylipins, see tables S12-S13. AA=arachidonic acid; $C G R P=c a l c i t o n i n$ gene related peptide; EpOME=epoxide of linoleic acid; H3=increased eicosapentaenoic acid (EPA) and docosahexaenoic acid (DHA); H3-L6=increased EPA+DHA, reduced linoleic acid; HETE=hydroxyeicosatetraenoic acid; HODE=hydroxyoctadecadienoic acid; LT=leukotriene; LTB4=leukotriene B4; PGE2=prostaglandin E2

increases in n-3 EPA and DHA, and several DHA derived antinociceptive oxylipins (including the primary biochemical endpoint 17-HDHA), and decreases in n-6 arachidonic acid and some pronociceptive oxylipins in circulation. Combined with the reduction in headache frequency and severity, these results support biological plausibility and suggest mechanisms underlying the observed pain reduction. Most notably, the H3 and H3-L6 diets increased EPA and DHA in circulating immune cells and erythrocytes, and increased plasma and serum concentrations of DHA derived oxylipins. These oxylipins are reported to have pain relieving properties, and serve as pathway markers and precursors for several families of lipid mediators with anti-inflammatory and antinociceptive properties, including resolvins, maresins, and n-3 monoepoxides. ${ }^{27} 29383967-70$

The observations that n-3 EPA and DHA in erythrocytes and DHA derived oxylipins in plasma are strongly inversely associated with headache hours is consistent with preclinical data showing potent antinociceptive effects of hydroxy-DHA derivatives, ${ }^{29-39}$ and our mechanistic model (fig 1). The positive association between erythrocyte linoleic acid content and headache hours is also consistent with our mechanistic model. Interestingly, despite diet induced decreases in arachidonic acid and 5-HETE in erythrocytes and plasma, respectively, neither arachidonic acid nor 5-HETE was associated with headache hours. A potential explanation for this lack of 

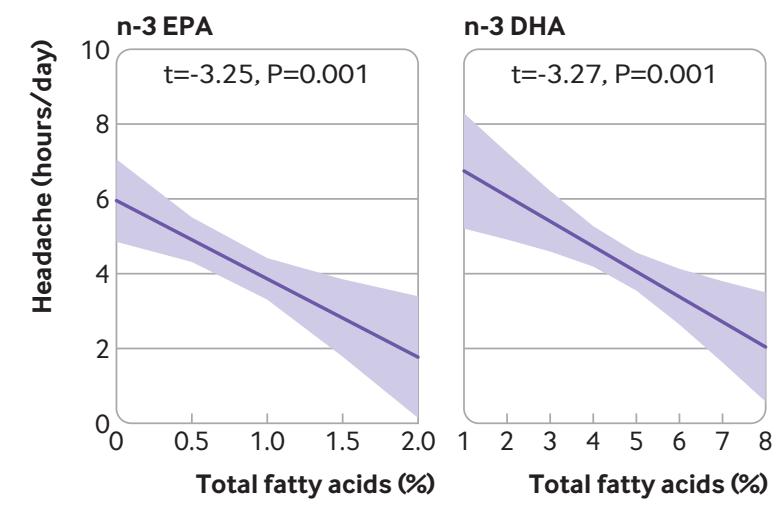

Precursor fatty acids in erythrocytes $(n=134)$
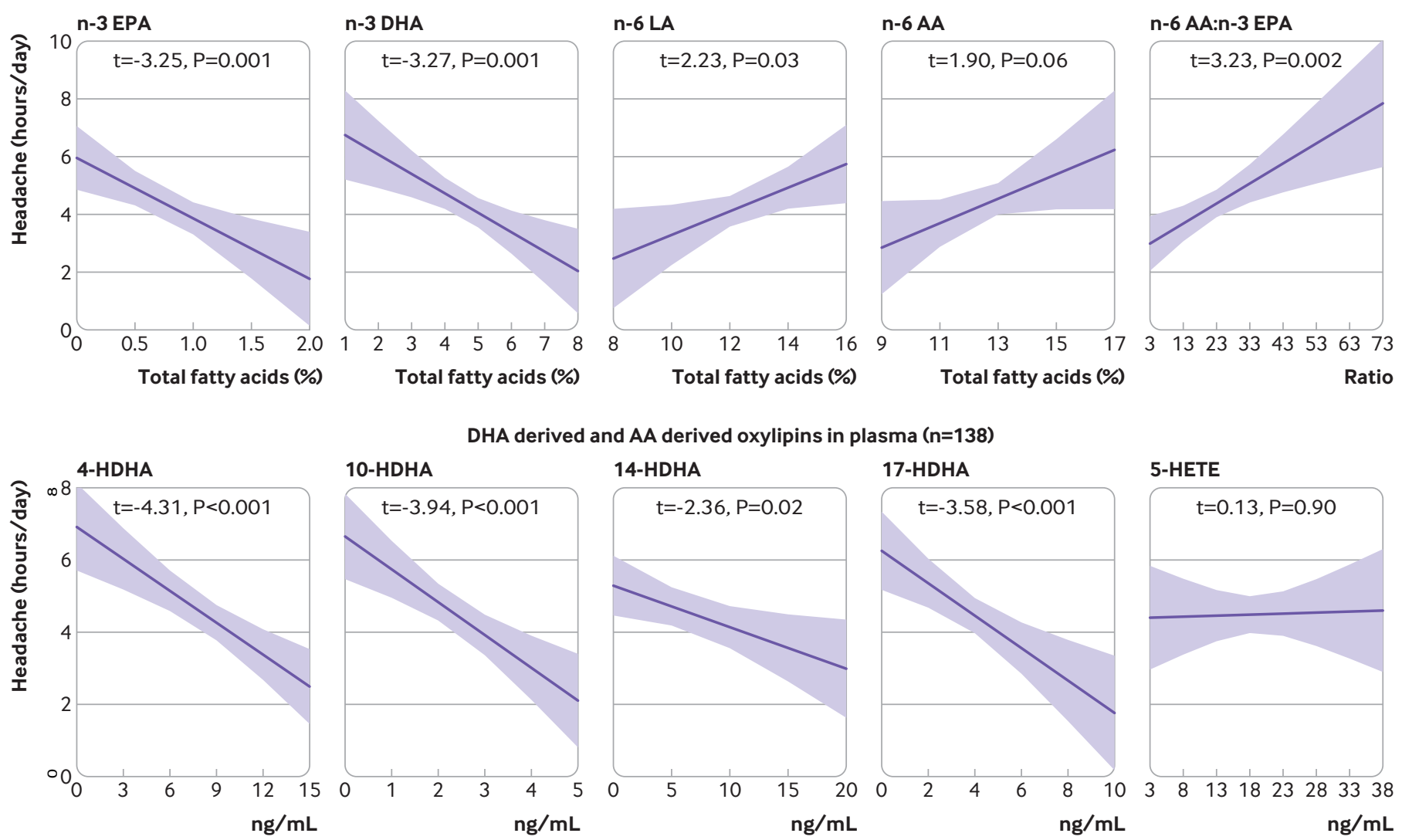

Fig 5 | Association of headache hours per day with precursor fatty acids and oxylipins (end of study). Each plot represents coefficients and $95 \%$ confidence intervals from linear regression model of targeted fatty acids and oxylipins on headache hours per day adjusted for the following baseline variables: age, body mass index, sex, headache hours per day, HIT-6, and chronic versus episodic migraine. Sample represents participants

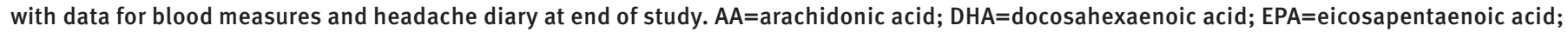
HDHA=docosahexaenoic acid; HETE=hydroxyeicosatetraenoic acid; HIT-6=six item headache impact test; LA=linoleic acid

association of headaches with arachidonic acid is that any benefit of lowering arachidonic acid was countered by the decreased NSAID or aspirin use by the H3-L6 group. However, because the arachidonic acid content of immune cells was not decreased in the active diets, it is also possible that changes in arachidonic acid and its derivatives are not driving pain reduction in the $\mathrm{H} 3$ and H3-L6 diets.

Arachidonic acid, prostaglandins, and leukotrienes Arachidonic acid is the precursor to several families of oxylipins that have been classically linked to immune activation and pain, including prostaglandins and leukotrienes. ${ }^{1471}$ Infusion of prostaglandins has been shown to evoke migraine like attacks in humans, ${ }^{21}$ ${ }^{22}$ and NSAIDs and aspirin targeting prostaglandin synthesis are widely used to treat acute headache pain. ${ }^{72}$ Leukotrienes are potent mediators of immune activation, which can initiate an inflammatory cascade in the sinuses and other tissues innervated by the trigeminal nerve, ${ }^{73}$ and have been indirectly linked to migraine pathogenesis. ${ }^{74}$ The H3 and H3L6 diets significantly decreased the arachidonic acid content of erythrocytes (but not immune cells). Interestingly, however, neither intervention affected concentrations of prostaglandin E2 measured by liquid chromatography tandem mass spectrometry and ELISA, or leukotriene B4 and cysteinyl leukotrienes measured by ELISA. NSAIDs and aspirin decrease pain by inhibiting conversion of arachidonic acid to 2-series prostaglandins. The H3-L6 group significantly decreased NSAID and aspirin use over the course of the intervention, which might have counteracted any diet induced reductions in prostaglandin E2. The H3 diet decreased 5-HETE, which serves as a pathway marker for 5-lipoxygenase mediated conversion of arachidonic acid to leukotrienes and lipoxins, ${ }^{75}$ and also has direct nociceptive actions in preclinical models. ${ }^{76}$ Other arachidonic acid derived eicosanoids with preclinical links to pain (eg, prostaglandin I2, lipoxins, hepoxilins) were not measured.

\section{Biochemical and clinical effects of dietary linoleic acid lowering}

The finding that the H3-L6 diet produced twice the reduction in headache days as the $\mathrm{H} 3$ diet is consistent with our hypothesis that lowering dietary linoleic acid could be a key component contributing to maximal pain reduction. We also observed that participants with lower linoleic acid concentration in blood had fewer headaches at end of study. A typical US diet has a linoleic acid intake of $7 \%$ of energy. We sought to 
decrease linoleic acid in the H3-L6 group to achieve intakes of $1.8 \%$ of energy (an amount consistent with human diets before the addition of concentrated vegetable oils), ${ }^{434}$ but achieved only a median intake of $3.2 \%$ of energy. This reduction in dietary linoleic acid decreased the linoleic acid content of erythrocytes and immune cells by $5-11 \%$, however it was not enough to decrease any of the linoleic acid derived oxylipins that we measured in circulation. Because the half life of linoleic acid in adipose tissue stores is estimated to be 680 days, ${ }^{77}$ a longer trial is needed to better understand the optimal degree and duration of dietary linoleic acid lowering. Additionally, such a trial could analyze the most relevant tissues, mediators, and mechanisms linking dietary linoleic acid lowering to clinical pain reduction.

\section{Strengths and limitations}

The strengths of the present study include the randomized controlled trial design, previously published protocol, use of a daily electronic diary designed to capture the presence and severity of headaches, controlled provision of foods and oils, and extensive biochemical analyses investigating mechanisms linking the interventions to pain. This study has several limitations that might affect interpretation and generalizability. Firstly, it is difficult to conduct tightly controlled diet intervention trials in free living populations, particularly to study the effects of nutrients with perceived health benefits such as n-3 fatty acids. The possibility exists that expectation of benefit from consuming foods rich in n-3 fatty acids contributed to the reported pain reduction. Similarly, the role of the unblinded dietitian in this study is a limitation. However, we did not find that participants' intervention credibility scores at baseline (after the first dietitian counselling session) were predictive of headache outcomes at end of study. Additionally, food provision was designed to equalize credibility across the interventions (supplement, page 3) and all endpoint assessments were conducted by blinded staff or reported directly by participants.

The choice of HIT- 6 as the primary outcome instead of a more specific measure of pain is a limitation of this study. The International Headache Society recommends the use of headache diary data for primary endpoints when assessing efficacy in chronic migraine populations. ${ }^{78}$ Unfortunately, we were not aware of these guidelines when the study was designed. Further, it is possible that the 16 week interventions were not long enough to produce improvement in traits, even in the presence of reduction in headache frequency and severity.

Linoleic acid was lowered in the H3-L6 intervention by using an isocaloric substitution with mostly monounsaturated fats and some saturated fats. The substitution of other nutrients in place of linoleic acid could yield different results. Despite the intensive nature of the interventions, we were unable to lower dietary linoleic acid to the $1.8 \%$ of energy target in the H3-L6 group. This highlights the challenge of decreasing linoleic acid in modern societies where linoleic acid enriched vegetable and seed oils are ubiquitous, and suggests a potential lack of generalizability of the H3-L6 diet findings to patients outside of a clinical research setting.

This study population was made up of 166 (88\%) women with a mean age of 38 years; therefore, the results are not necessarily generalizable to children, older adults, men, or other populations not represented in this trial. Additional limitations include the use of fasting, circulating fatty acids and oxylipins as a proxy for chronic exposures in tissues that might be more directly involved in headache pathogenesis (eg, cranial vessels, trigeminal nerve terminals), and the many limitations inherent in sample collection, processing, and analysis of oxylipins. ${ }^{79-81}$ More participants randomized to the H3-L6 diet used one category of preventive drugs (anticonvulsants) compared with participants randomized to the other groups. A sensitivity analysis adjusting for anticonvulsant use seemed to move estimates of clinical improvement further from the null (anticonvulsant use was associated with worse outcomes) and did not alter conclusions. While differences in baseline covariates can arise by chance, we also conducted a manual review of the randomization record and did not find any patterns indicating departure from the predesigned randomization scheme. Finally, this study was not powered for all the analyses presented and therefore wide confidence intervals are not unexpected. Larger, targeted studies will be needed to help clarify some of the suggestive but inconclusive findings.

\section{Future directions}

Steps for future research include testing the H3-L6 intervention in other populations with chronic pain; further clarifying mechanisms underlying physical pain reduction, including the effects of the interventions on additional nociceptive mediators derived from n-3 and n-6 fatty acids, and in tissues more directly implicated in headache pathogenesis; using statistical mediation analyses to identify relevant biochemical pathways and determine whether any nociceptive derivatives of n-3 and n- 6 fatty acids mediate the effect of diet group assignment on headache reduction; evaluating whether decreasing dietary linoleic acid to lower levels or administering the interventions for longer duration would result in greater pain reduction because turnover of polyunsaturated fatty acids in relevant tissues could take years. ${ }^{7782}$

\section{Conclusion}

We tested two active dietary interventions designed to target biochemical mechanisms known to regulate nociception. Both diets produced biochemical changes consistent with decreased nociception. While the diets did not significantly improve quality of life, they produced large, robust reductions in frequency and severity of headaches relative to the control diet. The H3-L6 diet was more effective than the $\mathrm{H} 3$ diet for some outcomes. This study provides a biologically plausible demonstration that pain can be treated through 
targeted dietary alterations in humans. Collective findings suggest causal mechanisms linking $n-3$ and n- 6 fatty acids to nociception, and open the door to new approaches for managing chronic pain in humans.

\section{AUTHOR AFFILIATIONS}

'Lipid Peroxidation Unit, Laboratory of Clinical Investigation, National Institute on Aging, Baltimore, MD, USA

${ }^{2}$ Intramural Program of the National Institute on Alcohol Abuse and Alcoholism, NIH, Bethesda, MD, USA

${ }^{3}$ Department of Physical Medicine and Rehabilitation, UNC School of Medicine, University of North Carolina at Chapel Hill, Chapel Hill, NC, USA

${ }^{4}$ Department of Psychiatry, UNC School of Medicine, University of North Carolina at Chapel Hill, Chapel Hill, NC, USA

${ }^{5}$ Metabolic and Nutrition Research Core, UNC Medical Center University of North Carolina at Chapel Hill, Chapel Hill, NC, USA

${ }^{6}$ Department of Medicine, UNC School of Medicine, University of North Carolina at Chapel Hill, Chapel Hill, NC, USA

${ }^{7}$ Cytokine Analysis Core, UNC Adams School of Dentistry, University of North Carolina at Chapel Hill, Chapel Hill, NC, USA

${ }^{8}$ Department of Perioperative Medicine, NIH Clinical Center, Bethesda, MD, USA

${ }^{9}$ Department of Biostatistics, Gillings School of Global Public Health, Chapel Hill, NC, USA

${ }^{10}$ Department of Neurology, UNC School of Medicine, University of North Carolina at Chapel Hill, Chapel Hill, NC, USA

The authors thank the study participants and acknowledge the following people for their research assistance: Ashley Harper (UNC Medical Center, Metabolic and Nutrition Research Core), Paula Anderson and Vania Wu (UNC Program on Integrative Medicine), and Carol Culver (UNC Cytokine Analysis Core). They acknowledge Marc Raley (Visual Media Services for intramural programs of NIDA and NIA) for the graphic art in figure 1 .

Contributors: CER and DZ contributed as first authors. CER led the conceptualization, design of the interventions and writing, and contributed to methodology, resources, and supervision. DZ led the statistical analysis, interpretation and writing, and contributed to the conceptualization, funding acquisition, and methodology. KRF led the funding acquisition, methodology administration, resources, and supervision of the trial, and contributed to the conceptualization, statistical analysis, and writing of the manuscript. BM led the design and administration of the dietary interventions and contributed to conceptualization and methodology. MH led the data curation, validation and visualization, and performed the statistical analysis. GSK, ZXY, and IL led the biochemical analyses. VM contributed to the statistical analysis. $\mathrm{CL}$ contributed to the project administration and resources. GH and JP supported the statistical analysis and methodology. RL, AFD, SMH, DAB, and AM supported the biochemical analyses. AJ supported the investigation. JRH supported the conceptualization and methodology. JMD contributed to the methodology and writing of the manuscript. OSP designed data collection instruments and contributed to the methodology. CMS contributed to the funding acquisition, statistical analysis, and methodology. SAG contributed to the conceptualization, funding acquisition, and resources. JDM acted as the study physician, led the conceptualization, funding acquisition, and contributed to supervision. All authors contributed to editing the manuscript. The corresponding author attests that all listed authors meet authorship criteria and that no others meeting the criteria have been omitted. CER, DZ, and KRF are guarantors.

Funding: This project was funded by grant 1R01AT007813-01A1 from the National Center for Complementary and Integrative Health (NCCIH), National Institutes of Health ( $\mathrm{NIH}$; main funding source). Additional support was provided by the intramural programs of the National Institute on Aging (NIA) and the National Institute on Alcohol Abuse and Alcoholism, NCCIH T32 Research Fellowship Program (T32 AT003378), the Mayday Fund, and the UNC Nutrition Obesity Research Center, CHAl Core (grant DK056350), National Institute of Diabetes and Digestive and Kidney Diseases (NIDDK), $\mathrm{NIH}$. We acknowledge the database support of the NC Translational and Clinical Sciences (NC TraCS) Institute, which is supported by the National Center for Advancing Translational Sciences (NCATS), NIH, through grant UL1TR002489. The funders had no role in considering the study design or in the collection, analysis, interpretation of data, writing of the report, or decision to submit the article for publication.
Competing interests: All authors have completed the ICMJE uniform disclosure form at www.icmje.org/coi_disclosure.pdf and declare: the $\mathrm{NCCIH}(\mathrm{NIH}), \mathrm{NIA}$, National Institute on Alcohol Abuse and Alcoholism, Mayday Fund, UNC Nutrition Obesity Research Center, CHAI Core, NIDDK (NIH), NC TraCS Institute, NCATS (NIH) for the submitted work; no financial relationships with any organizations that might have an interest in the submitted work in the previous three years. The NIA (NIH) has claimed intellectual property related to stable analogs of oxidized lipid mediators (PCT/US2018/041086) with this manuscript's coauthors, CER and GSK, named as inventors. All other authors declare no other relationships or activities that could appear to have influenced the submitted work.

The lead author affirms that this manuscript is an honest, accurate, and transparent account of the study being reported; that no important aspects of the study have been omitted; and that any discrepancies from the study as planned have been explained.

Dissemination to participants and related patient and public communities: Results will be disseminated through scientific publications and conference presentations.

Provenance and peer review: Not commissioned; externally peer reviewed

Ethical approval: Trial procedures were approved by the UNC Institutional Review Board (13-3284).

Data sharing: The data and code are available from the corresponding author upon reasonable request.

This is an Open Access article distributed in accordance with the Creative Commons Attribution Non Commercial (CC BY-NC 4.0) license, which permits others to distribute, remix, adapt, build upon this work non-commercially, and license their derivative works on different terms, provided the original work is properly cited and the use is noncommercial. See: http://creativecommons.org/licenses/by-nc/4.0/.

1 Burch RC, Loder S, Loder E, Smitherman TA. The prevalence and burden of migraine and severe headache in the United States: updated statistics from government health surveillance studies. Headache 2015;55:21-34. doi:10.1111/head.12482

2 Stovner Li, Hagen K, Jensen R, et al. The global burden of headache: a documentation of headache prevalence and disability worldwide. Cephalalgia 2007;27:193-210. doi:10.1111/j.14682982.2007.01288.x

3 Merikangas KR. Contributions of epidemiology to our understanding of migraine. Headache 2013:53:230-46. doi:10.1111/head.12038

4 Sastry PS. Lipids of nervous tissue: composition and metabolism. Prog Lipid Res 1985:24:69-176. doi:10.1016/0163-7827(85)90011-6

5 Ramsden CE, Ringel A, Majchrzak-Hong SF, et al. Dietary linoleic acid-induced alterations in pro- and anti-nociceptive lipid autacoids: implications for idiopathic pain syndromes?Mol Pain 2016;12. doi:10.1177/1744806916636386

6 Ramsden CE, Faurot KR, Zamora D, et al. Targeted alteration of dietary n-3 and n- 6 fatty acids for the treatment of chronic headaches: a randomized trial. Pain 2013;154:2441-51. doi:10.1016/j. pain.2013.07.028

Domenichiello AF, Sapio MR, Loydpierson AJ, et al. Molecular pathways linking oxylipins to nociception in rats. Pain 2021:22:275-99 doi:10.1016/j.jpain.2020.09.001

8 Ramsden CE, Ringel A, Feldstein AE, et al. Lowering dietary linoleic acid reduces bioactive oxidized linoleic acid metabolites in humans. Prostaglandins Leukot Essent Fatty Acids 2012;87:135-41. doi:10.1016/j.plefa.2012.08.004

9 Caligiuri SPB, Parikh M, Stamenkovic A, Pierce GN, Aukema HM. Dietary modulation of oxylipins in cardiovascular disease and aging. Am I Physiol Heart Circ Physiol 2017:313.H903-18, doi:10.1152/ ajpheart.00201.2017

10 Taha AY, Hennebelle M, Yang J, et al. Regulation of rat plasma and cerebral cortex oxylipin concentrations with increasing levels of dietary linoleic acid. Prostaglandins Leukot Essent Fatty Acids 2018;138:71-80. doi:10.1016/j.plefa.2016.05.004

11 Ramsden CE, Zamora D, Makriyannis A, et al. Diet-induced changes in $\mathrm{n}$-3-and $\mathrm{n}$-6-derived endocannabinoids and reductions in headache pain and psychological distress. J Pain 2015;16:707-16. doi:10.1016/i.jpain.2015.04.007

12 LaPaglia DM, Sapio MR, Burbelo PD, et al. RNA-Seq investigations of human post-mortem trigeminal ganglia. Cephalalgia 2018;38:91232. doi: $10.1177 / 0333102417720216$

13 Fujimoto N, Kaneko T, Eguchi N, Urade Y, Mizuno N, Hayaishi $\mathrm{O}$. Biochemical and immunohistochemical demonstration of a tightly bound form of prostaglandin E2 in the rat brain. Neuroscience 1992;49:591-606. doi:10.1016/03064522(92)90229-U

14 Osthues T, Sisignano M. Oxidized lipids in persistent pain states. Front Pharmacol 2019:10:1147. doi:10.3389/fphar.2019.01147 
15 Zimmer B, Angioni C, Osthues T, et al. The oxidized linoleic acid metabolite 12,13-DiHOME mediates thermal hyperalgesia during inflammatory pain. Biochim Biophys Acta Mol Cell Biol Lipids 2018;1863:669-78. doi:10.1016/j.bbalip.2018.03.012

16 Hargreaves KM, Ruparel S. Role of oxidized lipids and TRP channels in orofacial pain and inflammation. J Dent Res 2016;95:1117-23. doi:10.1177/0022034516653751

17 Ruparel S, Hargreaves KM, Eskander M, et al. Oxidized linoleic acid metabolite-cytochrome P450 system (OLAM-CYP) is active in biopsy samples from patients with inflammatory dental pain. Pain 2013;154:2363-71. doi:10.1016/j.pain.2013.07.011

18 Patwardhan AM, Akopian AN, Ruparel NB, et al. Heat generates oxidized linoleic acid metabolites that activate TRPV1 and produce pain in rodents. J Clin Invest 2010;120:1617-26. doi:10.1172/ $\mathrm{JCl} 41678$

19 Patwardhan AM, Scotland PE, Akopian AN, Hargreaves KM. Activation of TRPV1 in the spinal cord by oxidized linoleic acid metabolites contributes to inflammatory hyperalgesia. Proc Natl Acad Sci U S A 2009;106:18820-4. doi:10.1073/pnas.0905415106

20 Ramsden CE, Domenichiello AF, Yuan ZX, et al. A systems approach for discovering linoleic acid derivatives that potentially mediate pain and itch. Sci Signal 2017;10:eaal5241. doi:10.1126/scisignal. aal5241

21 Antonova M, Wienecke T, Olesen J, Ashina M. Prostaglandin $\mathrm{E}(2)$ induces immediate migraine-like attack in migraine patients without aura. Cephalalgia 2012;32:822-33. doi:10.1177/0333102412451360

22 Wienecke T, Olesen J, Ashina M. Prostaglandin I2 (epoprostenol) triggers migraine-like attacks in migraineurs. Cephalalgia 2010;30:179-90. doi:10.1111/j.1468 2982.2009.01923.x

23 Doolen S, Keyes GS, Ramsden CE. Hydroxy-epoxide and keto-epoxide derivatives of linoleic acid activate trigeminal neurons. Neurobiol Pain 2020;7:100046. doi:10.1016/j.ynpai.2020.100046

24 Green D, Ruparel S, Gao X, et al. Central activation of TRPV1 and TRPA1 by novel endogenous agonists contributes to mechanical allodynia and thermal hyperalgesia after burn injury. Mol Pain 2016. doi:10.1177/1744806916661725

25 Green DP, Ruparel S, Roman L, Henry MA, Hargreaves KM. Role of endogenous TRPV1 agonists in a postburn pain model of partial-thickness injury. Pain 2013;154:2512-20. doi:10.1016/j. pain.2013.07.040

26 Xu ZZ, Liu XJ, Berta T, et al. Neuroprotectin/protectin D1 protects against neuropathic pain in mice after nerve trauma. Ann Neurol 2013;74:490-5. doi:10.1002/ana.23928.

27 Xu ZZ, Zhang L, Liu T, et al, Resolvins RvE1 and RvD1 attenuate inflammatory pain via central and peripheral actions. Nat Med 2010;16:592-7. doi:10.1038/nm.2123

28 Park CK, Xu ZZ, Liu T, Lü N, Serhan CN, Ji RR. Resolvin D2 is a potent endogenous inhibitor for transient receptor potential subtype V1/ A1, inflammatory pain, and spinal cord synaptic plasticity in mice: distinct roles of resolvin D1, D2, and E1. J Neurosci 2011;31:184338 doi:10.1523/INEUROSCI.4192-11.2011

29 Morisseau C, Inceoglu B, Schmelzer K, et al. Naturally occurring monoepoxides of eicosapentaenoic acid and docosahexaenoic acid are bioactive antihyperalgesic lipids. J Lipid Res 2010;51:3481-90. doi:10.1194/jlr.M006007

30 Ikeda A, Muroki A, Suzuki C, Shimazu Y, Takeda M. Resolvin D1 suppresses inflammation-induced hyperexcitability of nociceptive trigeminal neurons associated with mechanical hyperalgesia. Brain Res Bull 2020;154:61-7. doi:10.1016/j.brainresbull.2019.11.002

31 Bang S, Yoo S, Yang TJ, Cho H, Kim YG, Hwang SW. Resolvin D1 attenuates activation of sensory transient receptor potential channels leading to multiple anti-nociception.Br J Pharmacol 2010;161:70720. doi:10.1111/j.1476-5381.2010.00909.x

32 Bang S, Yoo S, Yang TJ, Cho H. Hwang SW. 17(R)-resolvin D1 specifically inhibits transient receptor potential ion channel vanilloid 3 leading to peripheral antinociception. BrJ Pharmacol 2012;165:683-92. doi:10.1111/j.14765381.2011.01568.x

33 Xu ZZ, Berta T, Ji RR. Resolvin E1 inhibits neuropathic pain and spinal cord microglial activation following peripheral nerve injury. I Neuroimmune Pharmacol 2013;8:37-41. doi:10.1007/s11481 012-9394-8

34 Klein CP, Sperotto ND, Maciel IS, Leite CE, Souza AH, Campos MM. Effects of D-series resolvins on behavioral and neurochemical changes in a fibromyalgia-like model in mice. Neuropharmacology 2014;86:57-66. doi:10.1016/j. neuropharm.2014.05.043

35 Jo YY, Lee JY, Park CK. Resolvin E1 inhibits Substance P-induced potentiation of TRPV1 in primary sensory neurons. Mediators Inflamm 2016. doi:10.1155/2016/5259321

36 Fonseca FC, Orlando RM, Turchetti-Maia RM, de Francischi IN. Comparative effects of the $\omega 3$ polyunsaturated fatty acid derivatives resolvins $\mathrm{E} 1$ and D1 and protectin DX in models of inflammation and pain. J Inflamm Res 2017;10:119-33. doi:10.2147/JIR.S142424

37 Park CK. Maresin 1 Inhibits TRPV1 in temporomandibular jointrelated trigeminal nociceptive neurons and TMJ inflammationinduced synaptic plasticity in the trigeminal nucleus. Mediators Inflamm 2015. doi:10.1155/2015/275126

38 Fattori V, Pinho-Ribeiro FA, Staurengo-Ferrari L, et al. The specialised pro-resolving lipid mediator maresin 1 reduces inflammatory pain with a long-lasting analgesic effect. Br J Pharmacol 2019;176:172844. doi:10.1111/bph.14647

39 Valdes AM, Ravipati S, Menni C, et al. Association of the resolvin precursor 17-HDHA, but not D- or E- series resolvins, with heat pain sensitivity and osteoarthritis pain in humans. Sci Rep 2017;7:10748. doi:10.1038/s41598-017-09516-3

40 Ramsden CE, Hennebelle M, Schuster S, et al. Effects of diets enriched in linoleic acid and its peroxidation products on brain fatty acids, oxylipins, and aldehydes in mice. Biochim Biophys Acta Mol Cell Biol Lipids 2018;1863:1206-13. doi:10.1016/j. bbalip.2018.07.007

41 Ramsden CE, Faurot KR, Zamora D, et al. Targeted alterations in dietary $n-3$ and $n-6$ fatty acids improve life functioning and reduce psychological distress among patients with chronic headache: a secondary analysis of a randomized trial. Pain 2015:156:587-96. doi:10.1097/01.j.pain.0000460348.84965.47

42 MacIntosh BA, Ramsden CE, Faurot KR, et al. Low-n-6 and low-n-6 plus high-n-3 diets for use in clinical research. $\mathrm{Br}$ J Nutr 2013;110:559-68. doi:10.1017/S0007114512005181

43 Blasbalg TL, Hibbeln JR, Ramsden CE, Majchrzak SF, Rawlings RR. Changes in consumption of omega-3 and omega- 6 fatty acids in the United States during the 20th century. Am J Clin Nutr 2011;93:95062. doi:10.3945/ajcn.110.006643

44 Ramsden CE, Zamora D, Majchrzak-Hong S, et al. Re-evaluation of the traditional diet-heart hypothesis: analysis of recovered data from Minnesota Coronary Experiment (1968-73). BMJ 2016;353:i1246. doi:10.1136/bmj.i1246

45 Mann JD, Faurot KR, Maclntosh B, et al. A sixteen-week threearmed, randomized, controlled trial investigating clinical and biochemical effects of targeted alterations in dietary linoleic acid and n-3 EPA+DHA in adults with episodic migraine: Study protocol. Prostaglandins Leukot Essent Fatty Acids 2018;128:41-52. doi:10.1016/j.plefa.2017.11.002

46 Maclntosh BA, Ramsden CE, Honvoh G, et al. Methodology for altering omega-3 EPA+DHA and omega-6 linoleic acid as controlled variables in a dietary trial. Clin Nutr 2021. doi:10.1016/j. clnu.2021.04.050

47 Devilly GJ, Borkovec TD. Psychometric properties of the credibility/ expectancy questionnaire. J Behav Ther Exp Psychiatry 2000;31:7386. doi:10.1016/S0005-7916(00)00012-4

48 Borkovec TD, Nau SD. Credibility of analogue therapy rationales. Behav Ther Exp Psy 1972;3:257-60. doi:10.1016/0005 7916(72)90045-6.

49 Curtis P, Gaylord SA, Park J, et al. Credibility of low-strength static magnet therapy as an attention control intervention for a randomized controlled study of CranioSacral therapy for migraine headaches. J Altern Complement Med 2011;17:711-21. doi:10.1089/ acm.2010.0277

50 Kawata AK, Coeytaux RR, Devellis RF, Finkel AG, Mann JD, Kahn K. Psychometric properties of the HIT-6 among patients in a headachespecialty practice. Headache 2005;45:638-43. doi:10.1111/j.1526$4610.2005 .05130 x$

51 Kosinski M, Bayliss MS, Bjorner JB, et al. A six-item short-form survey for measuring headache impact: the HIT-6. Qual Life Res 2003;12:963-74. doi:10.1023/A:1026119331193

52 Smelt AF, Assendelft WJ, Terwee CB, Ferrari MD, Blom JW. What is a clinically relevant change on the HIT-6 questionnaire? An estimation in a primary-care population of migraine patients. Cephalalgia 2014;34:29-36. doi:10.1177/0333102413497599

53 Headache Classification Committee of the International Headache Society (IHS). Headache Classification Committee of the International Headache Society (IHS) The International Classification of Headache Disorders, 3rd edition. Cephalalgia 2018;38:1-211. doi:10.1177/0333102417738202

54 Yuan ZX, Majchrzak-Hong S, Keyes GS, Iadarola MJ, Mannes AJ, Ramsden CE. Lipidomic profiling of targeted oxylipins with ultra performance liquid chromatography-tandem mass spectrometry. Anal Bioanal Chem 2018;410:6009-29. doi:10.1007/s00216-0181222-4

55 Ostermann Al, Koch E, Rund KM, Kutzner L, Mainka M, Schebb NH. Targeting esterified oxylipins by LC-MS - Effect of sample preparation on oxylipin pattern. Prostaglandins Other Lipid Mediat 2020;146:106384. doi:10.1016/j. prostaglandins.2019.106384

56 Stata Statistical Software. Release 16. [program] StataCorp LLC, 2019. 
57 Cameron AC, Trivedi PK. Regression analysis of count data. Second edition. Cambridge University Press, 2013. doi:10.1017/ CB09781139013567

58 Herd CP, Tomlinson CL, Rick C, et al. Cochrane systematic review and meta-analysis of botulinum toxin for the prevention of migraine. $B M$ J Open 2019:9:e027953. doi:10.1136/bmjopen-2018-027953.

59 Lattanzi S, Brigo F, Trinka E, et al. Erenumab for preventive treatment of migraine: a systematic review and meta-analysis of efficacy and safety. Drugs 2019:79:417-31. doi:10.1007/s40265-019-01069-1.

60 Burch RC, Buse DC, Lipton RB. Migraine: epidemiology, burden, and comorbidity. Neurol Clin 2019;37:631-49. doi:10.1016/j. ncl.2019.06.001.

61 Burch R, Rizzoli P, Loder E. The prevalence and impact of migraine and severe headache in the United States: Updated age, sex, and socioeconomic-specific estimates from government health surveys. Headache 2021:61:60-8 doi:10.1111/head.14024

62 D’Antona L, Matharu M. Identifying and managing refractory migraine: barriers and opportunities?J Headache Pain 2019;20:89. doi:10.1186/s10194-019-1040-x

63 Chai NC, Bond DS, Moghekar A, Scher Al, Peterlin BL. Obesity and headache: Part II--potential mechanism and treatment considerations. Headache 2014:54:459-71. doi:10.1111/head.12297

64 Chai NC, Scher Al, Moghekar A, Bond DS, Peterlin BL. Obesity and headache: part I--a systematic review of the epidemiology of obesity and headache. Headache 2014;54:219-34. doi:10.1111/head.12296

65 Schwedt TJ, Hentz JG, Sahai-Srivastava S, et al, MOTS Investigators. Headache characteristics and burden from chronic migraine with medication overuse headache: cross-sectional observations from the Medication Overuse Treatment Strategy trial. Headache 2021;61:351-62. doi:10.1111/head.14056

66 Lands WE, Libelt B, Morris A, et al. Maintenance of lower proportions of (n-6) eicosanoid precursors in phospholipids of human plasma in response to added dietary (n-3) fatty acids. Biochim Biophys Acta 1992;1180:147-62. doi:10.1016/0925-4439(92)90063-S

67 Domenichiello AF, Jensen JR, Zamora D, et al. Identifying oxidized lipid mediators as prognostic biomarkers of chronic posttraumatic headache. Pain 2020;161:2775-85. doi:10.1097/j. pain.0000000000001983

68 Zhang L, Terrando N, Xu ZZ, et al. Distinct analgesic actions of DHA and DHA-derived specialized pro-resolving mediators on post-operative pain after bone fracture in mice. Front Pharmacol 2018;9:412. doi:10.3389/fphar.2018.00412

69 Luo X, Gu Y, Tao X, Serhan CN, Ji RR. Resolvin D5 inhibits neuropathic and inflammatory pain in male but not female mice: distinct actions of D-series resolvins in chemotherapy-induced peripheral neuropathy. Front Pharmacol 2019;10:745. doi:10.3389/ fphar.2019.00745

70 Barden AE, Moghaddami M, Mas E, Phillips M, Cleland LG, Mori TA Specialised pro-resolving mediators of inflammation in inflammatory arthritis. Prostaglandins Leukot Essent Fatty Acids 2016;107:24-9. doi:10.1016/j.plefa.2016.03.004

71 Jang Y, Kim M, Hwang SW. Molecular mechanisms underlying the actions of arachidonic acid-derived prostaglandins on peripheral nociception. J Neuroinflammation 2020;17:30. doi:10.1186/ s12974-020-1703-1

72 Tepper SJ. Acute treatment of migraine. Neurol Clin 2019;37:727-42. doi:10.1016/j.ncl.2019.07.006

73 Parnes SM. Targeting cysteinyl leukotrienes in patients with rhinitis, sinusitis and paranasal polyps. Am J Respir Med 2002;1:403-8. doi:10.1007/BF03257167

74 Ince H, Aydin OF, Alaçam H, Aydin T, Azak E, Özyürek H. Urinary leukotriene $\mathrm{E} 4$ and prostaglandin $\mathrm{F} 2 \mathrm{a}$ concentrations in children with migraine: a randomized study. Acta Neurol Scand 2014;130:188-92. doi:10.1111/ane.12263.

75 Serhan CN. On the relationship between leukotriene and lipoxin production by human neutrophils: evidence for differential metabolism of 15-HETE and 5-HETE. Biochim Biophys Acta 1989;1004:158-68. doi:10.1016/0005-2760(89)90264-6

76 Hwang SW, Cho H, Kwak J, et al. Direct activation of capsaicin receptors by products of lipoxygenases: endogenous capsaicinlike substances. Proc Natl Acad Sci U S A 2000;97:6155-60. doi:10.1073/pnas.97.11.6155

77 Dayton S, Hashimoto S, Dixon W, Pearce ML. Composition of lipids in human serum and adipose tissue during prolonged feeding of a diet high in unsaturated fat. / Lipid Res 1966;7:103-11. doi:10.1016/ S0022-2275(20)39591-2

78 Tfelt-Hansen P, Pascual J, Ramadan N, et al, International Headache Society Clinical Trials Subcommittee. Guidelines for controlled trials of drugs in migraine: third edition. A guide for investigators. Cephalalgia 2012;32:6-38. doi:10.1177/0333102411417901

79 Liakh I, Pakiet A, Sledzinski T, Mika A. Modern methods of sample preparation for the analysis of oxylipins in biological samples. Molecules 2019;24:1639. doi:10.3390/molecules24081639

80 Hartung NM, Mainka M, Kampschulte N, Ostermann Al, Schebb NH. A strategy for validating concentrations of oxylipin standards for external calibration. Prostaglandins Other Lipid Mediat 2019;141:22-4. doi:10.1016/j.prostaglandins.2019.02.006

81 Ramsden CE, Yuan ZX, Horowitz MS, et al. Temperature and time-dependent effects of delayed blood processing on oxylipin concentrations in human plasma. Prostaglandins Leukot Essent Fatty Acids 2019;150:31-7. doi:10.1016/j.plefa.2019.09.001

82 Umhau JC, Zhou W, Carson RE, et al. Imaging incorporation of circulating docosahexaenoic acid into the human brain using positron emission tomography. J Lipid Res 2009;50:1259-68. doi:10.1194/jlr.M800530-JLR200

\section{Web appendix: Supplement}

